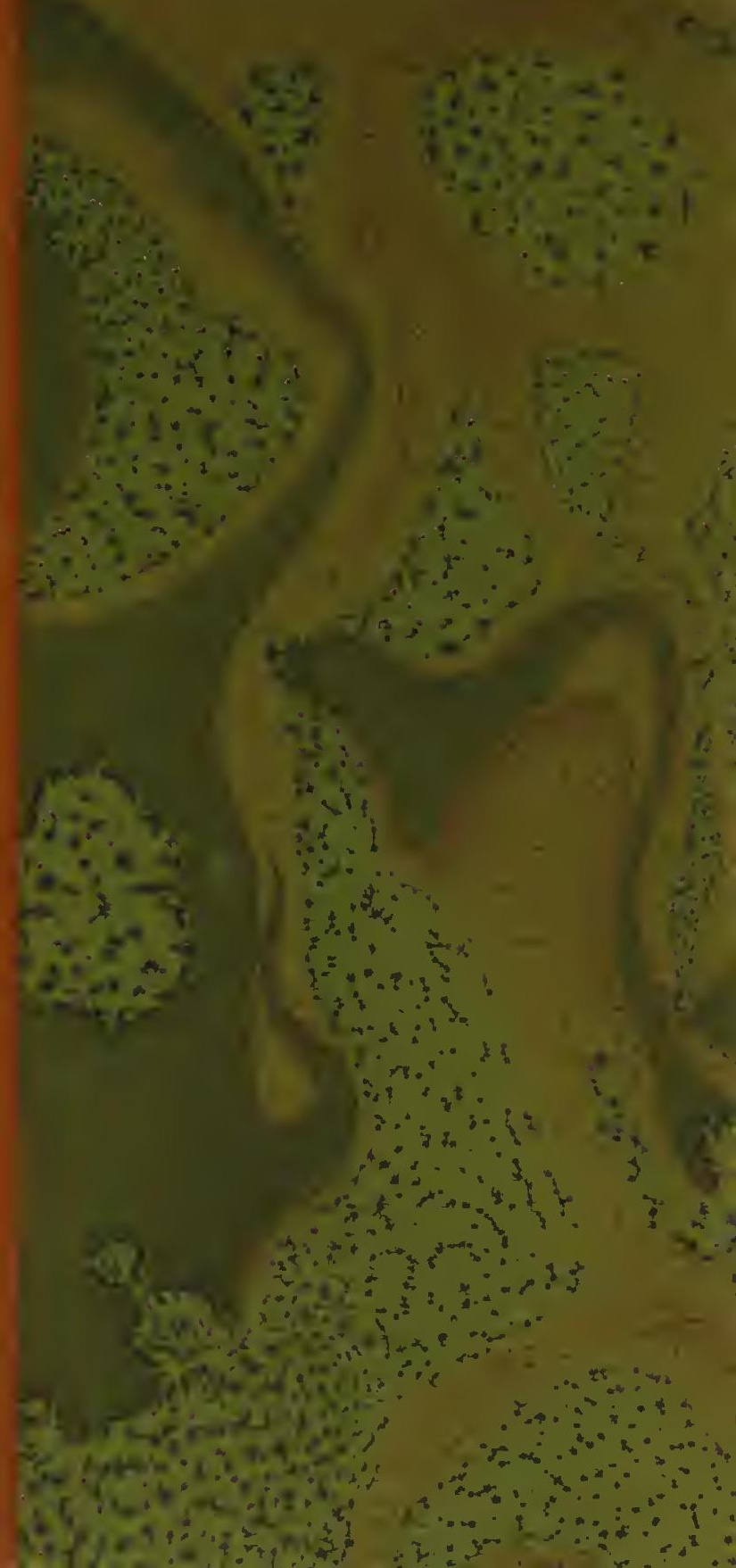













\section{Det solffíantige}

\section{$\mathfrak{b} \mathfrak{i} \mathfrak{e} \mathfrak{e} \mathfrak{n}=\mathfrak{W} \mathfrak{A} \mathfrak{x} \mathfrak{t} \mathfrak{e}$ \\ odec}

Rủktict)e 2nnotifungen

ระ

$+$

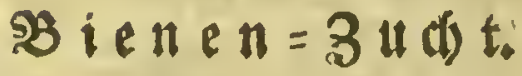

3ufamtnen getragen auß ben Sduriften bon Ehrif: Columella, 2 beit und M?. Garafti.

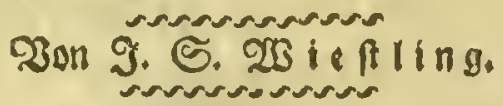

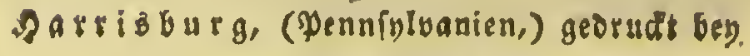

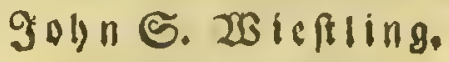

1819. 
Eastern District of Pennsylvania, to wit :

BE IT REMEMBERED, that on the TENTH DAY OF MAY, in the FORTYTTHUD year of the INDEPENDENCE of the UNITED STA'TES of America, A. D. 1819. JOHN S. WILisl'LING, of the said district, hath deposited in this office the Title of a book the right whereof he claims as author, in the words following, to wit:

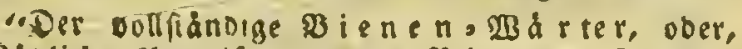

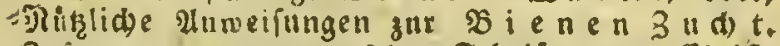
3ufummen getragen aub ben Sdriften von Egrift,

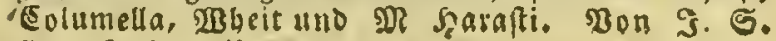
"WB t e fit in $g . "$

In conformity to the act of the Congress of the United States, intituled, "An Act, for the encouragement of learning, by securing the copies of Maps, Charts, and Books, to the authors and proprietors of such co'pies, during the times therein Mentioned." - And also to the Act, entitled, "An Act supplementary to an 'act, entitled, "An Act for the elicouragement of lear"ning, by securing the copies of Maps, Charts, and "Books, to the authors and proprietors of such copies "luring the times therein mentioned." and extending the benefits thereof to the arts of designing, engraving, and etching listorical and other prints."

D. CALDWELI, Clk. of the eastern district, of Penna.

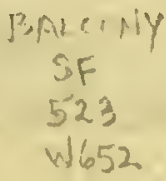



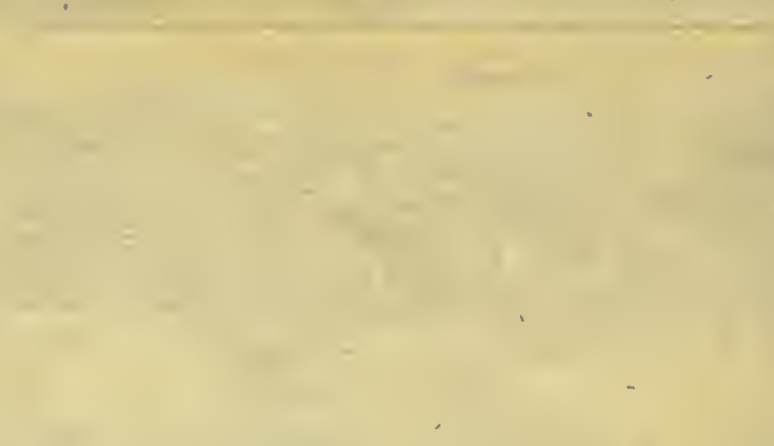

=

- 



\section{Borbericht}

Pin bie gencigten tno geefrten Esfer.

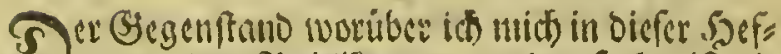
te nn Daş \$ublieum zu inenden fucbse, ift cis ner, Der buted eine gefdicftere Feoce als die Meis nige iff, auscinanoes gefest zu werden verbienct. (Dod) noer bin id uberkengt, onf, wenn einiger meiner Sefen, Die 2Inweifungen befolgt, Die in

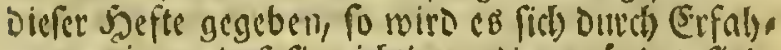

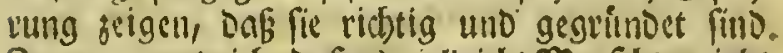
Broar erwarte ids: Daf es viellecidt Menf(hen gicbt,

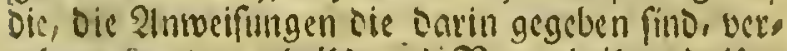

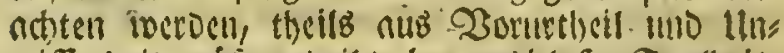
miffenleit, grégtentheciss aber sus blo fer Fonuliscit.

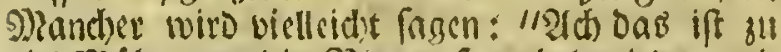
viel gribe, um die Bienen fo gu belsanden, wie uns lice ancmpfoblen wiro, ons thue ich) nicht, es ift nid)t notlswenoig Daf man mit ilinen fo vore fichtig ift, fie thun am beften menn man gar nichto an ilynen thut." Soldben mollte idh blus fangen,

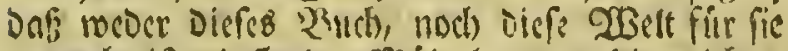

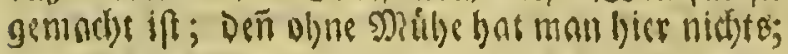


uno forthe, bie firls olyne glílye Duret) zu bringen fuchen, werden nur felse wenig SButter baben ithre Suppen zul filymelsen, Doer Jgonig in il)een Saffee

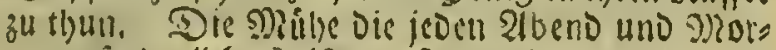
gen erioroerfich ift, ift nutr felse gering uno fann in ein Эona. Dinuten getlan werben; und we

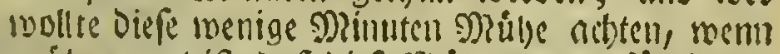

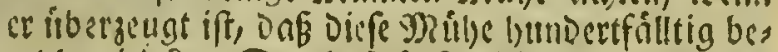

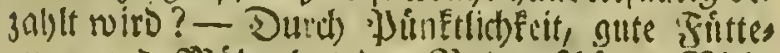

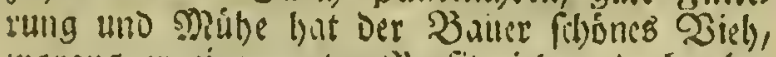
svoralts er cinen guten 'Jrofit zielset; Doth aber g!nube ids nid)t, onf er von feinem $\mathfrak{B i e l}$ (in $\mathfrak{Z} e r$ báltnif) fo vidr veinen Đrofit hat, mit fo wenig

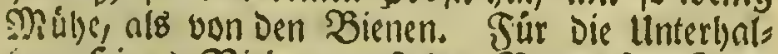

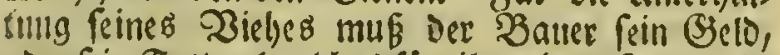
soer fein Futter (weld)es fúr ilgn eben fo gut ift als (Sjelo) anmenden, woeldes ber ben SBienen Der Fall nicht ît ; diefé ernályeren fich felber; fie famm: len ibre Pabrung, nebft Dem \$rofit ilgres Eigncre, yon Den Slíttern uno Blumen, aus weleten Der gútige Sdjopfer Der Ratur befetlog Daß fie ilje Talbenng bleben follten.- Seit verfdjiedenen galy=

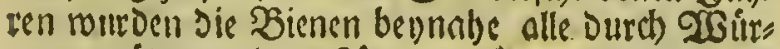
iner getodet uno zerfturt, mefeldes nid)t allein sul folchen die fie batten, cir: phibat, fondern anth eir

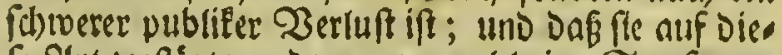

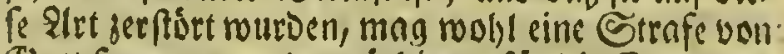
Soott feun, um uns zu nothigen, fin Die Erbaltung Der colen Girfelten, welche er fúr Den Nuken feiner Menfden=sinder fduf, unfere (S) Donfen onf cine nnoere 2lrt, fie ju bebnnolen, jul renfen. Seit

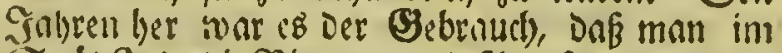

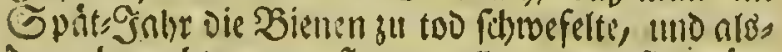
senn berauble man fee vou nllem mas fie boibs 
reno bem Sommer fammelten. Rann man fich etrons araufameres onten als Diefes; Den gans zen Sommer arbeiten Die Bisnen, uno fír went 9iid)t für ihre blofe Uliterl)altung. Reili, fondern fiit' Den Puken Der Menfden, uno Dennodt) wers den. fie in Spitjabr, von Denen, fúr wolde fie voil) rend ibrem ganzen \&cbon arbeiten, mit Feuce und Ed)wefil, obme einiges Giefillir Des Di itcioz alle zerfturt. Wielleidt miro mandber frach:

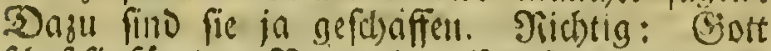

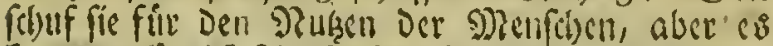

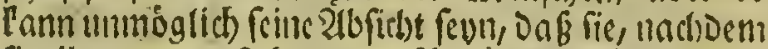
fie il)r ganzes \&ebentang firr Die $\mathbb{R e n}$ (d)en gear beitst haben, von ibnen graufamlid) bingerthbtet worden follten, um depto beffer im Stande zu fenn fie zu berauben. Nit Dem nemlichent Diecht, fén, te eill Sdjăfer allen feinen Sabaafen Die Sadrfe

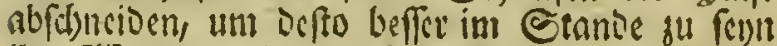

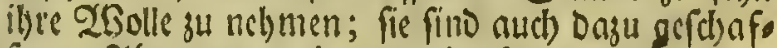
fen. 2lber wom cinmal ein Mann Diefse thun wirros, wintoe nicht ein Geoerfagen: Disfer ift

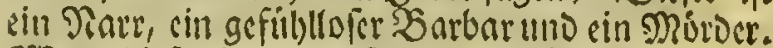

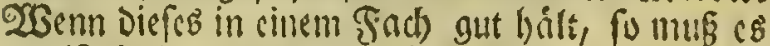
getwis in Dem andern aud beffelsell. Die 916 .

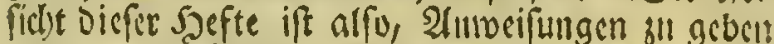

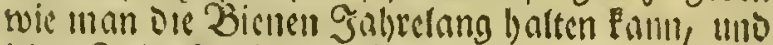

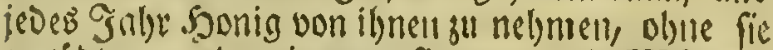
3u tobten; unt wis man fie gegen Dic 20 Birmer

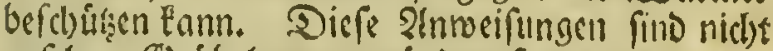

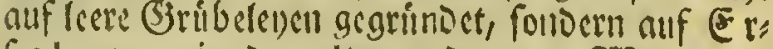
fabrullg in Der nifen uno neuen 28 elt.

E\& war zuerft beabfictete Dof cin after torirois get Buirger yon Dauplin (Eauty), mit mir in

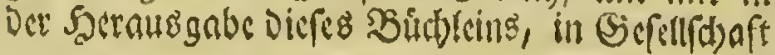




\section{(6)}

gethen swollte; weil er aber nid)t twilleno war feinen Эamen als 2(ut) or, bor Das Đublifum Fommen zu laffen, fo siberlies er mir Die ganze Sad)e, gab mir jeDod) alle mogliche unterftitsung, und folg,

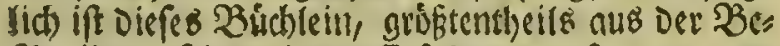
fdyecibung feiner eignen (Erfal)rung zufammen ges rezt, theif(s aber alth aub Den Sd)rciben bon

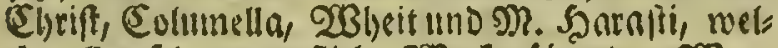

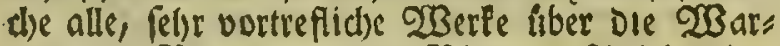
tung und Bebnnolung Der Bienen geftreieben bas ben.

$$
\text { Э. ธ. } 22 .
$$

ज马arrizbutg, Den 28 ften 2tpril 1819. 


\section{Anteifung}

oser Lurger Unterrictst wie man bie Bienen gegen nlle ibre Fe in be erbalten finnn.

arion

$\mathfrak{B}$

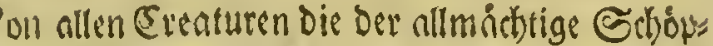
fer erfechaffen bnt, ift feine fo col, und nud Eeis tle Die weniger Sdaben thut nib vie Biene. 2(tid)

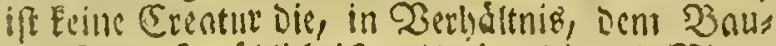
eloss? roelun er nur wifte wie er Dicfelben gegen ibre Feinoe foubsen fónnte.

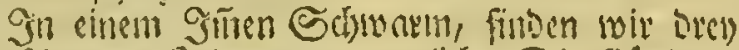
verfuciere Sronungen, nemlid): Die Sionigin, Die 23 rut = Smen und Die Eshnff: Sñen oder $21 \%$ bciter. Dielucil $\mathrm{CE}$ nber nicht fo ununginglid)

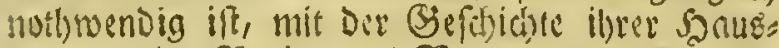

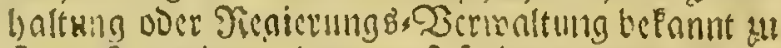

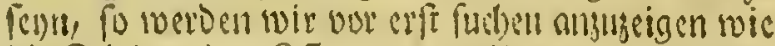
Die Feinoen Der Gutren z! vertilgen und nbzulgn!s ten fino; แno abอง

\section{Bon den Jeinden Der Sienen.}

Die Freinde welche dic Dicnen lsaben, fino man dber Siet und $e^{3}$ iff Desinegen nothioendig fie zu nennen, und Die গुelgandungen bel) Der Berfts: rang ooce 2 bboltung cines jeden foleben feinoes, beviufügen.

(5) fteng.-Die Epinne ift ibnen cin srober

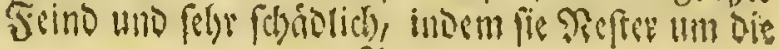
अ 2 
Strinde weben, worin die Bienen vef, und Dann Durch ie Sopinnea gefangen werden. Llm fie zu

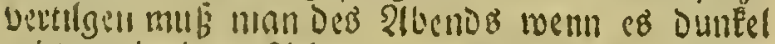
suiro, mit sinem sidft an Den Stano gehen, Dem Das ift ibere Zeit Daf fie an Den Eingang Der Sim men gel)en um auf fie zu fatcen, uno nlobenn fann man lie leicht toden.

Z 1 e 1) te 118 . Die Ameifen fino Den Bienen fo berlyabit, Daf man felten sthe Biene fieht eine

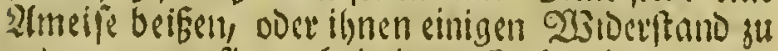
geben, weinl fic aucl) in ilyeen siorb bringen, mos

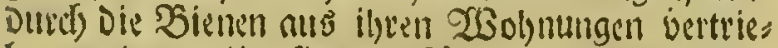

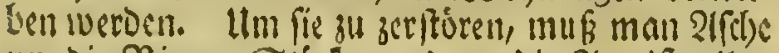
"Im die SBienten Eturfe, uno soo die ?tmeifen ilyre Bsinge baben, berum ftreuen, oder man Eann aluid) Sald netymen, aber Diefer ift Den Bienen fibiolid), wenn er etron in die Rórbe fummen folls te; Der fickerfie und befte $28 \mathrm{eg}$ itt aber, recun man ausfinden famn too fie ilye Refter baben, und fockeno 230 fifer nimmt uno fie ful tode brits bet.

Drittens-Dar Gmmen. Frab ift ihnen ferter

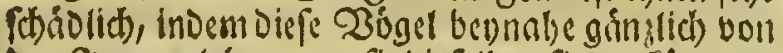
Den Immenleben, too fie Dicfelben finden Éounen; Destbegen follte man futben ibere Refter zu zerffs: ven und Die 2llten zll tóden auf jede sirt wie mati ihnen beytommen lann.

Z ierten8.-Dbfebon die rsurmes, oder die Fleoess Mdufe gulest gemeloet werden, fo find fie Dennoch die grósten Feinde roelche die sienen

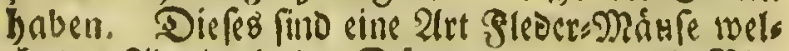
the Des abends in Der Dasmmetung, an de SBie. nen-2 srbe ge!en, in Diefelben Gimeinfriedten, und igren Sanmen in die leeren Diofen, (roenn fie fols 


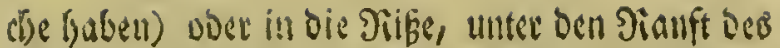
Rurbes ooer fonft irgendono binlegen; und un cttis

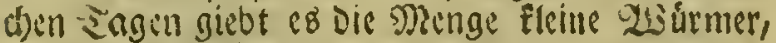

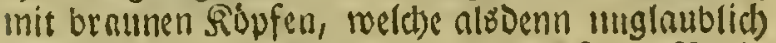

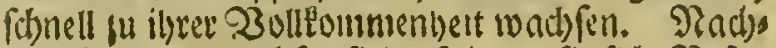
Dem fie allogetwad)fen find, fwinnen fie fich Drefter auf Die Itrt wie SpinnemsRefter, worin Die Bies nen feft werden; Heriechen albDenn wiberziperg Durd) Die Siofen, onß Der Şonig allblatft, und tóden aud) Den SBienen ilyre Brut (oder Yungen) Iocls the alsoem in grofer solenge (weenn Die sBienen

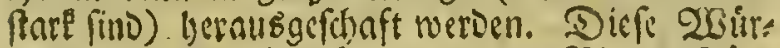

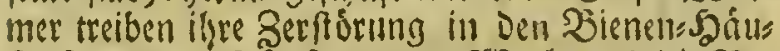

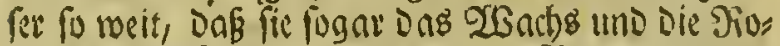
fen cilles auffresen und treiben die Dienen aus ib) rer Sheimatly. SGenn man Deb 2(bende bel) Esı1. nen lintergang an Den Bienen: Stuno gelset, fo

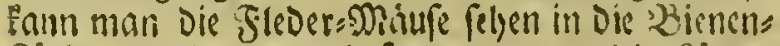
Rutbe lineingeben, befunders menn die fóci)er

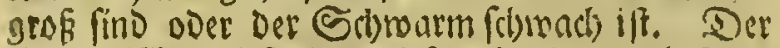
einzige 23 eg Defes llngegiefer abzuhalten, befrebet Darin, Daf man den Bbienen folibe 250 olyungen giebt, worin forches lungegicfer feinen Sillfenthalt

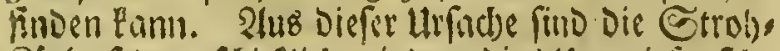

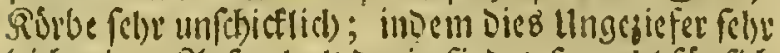
licibt einen 2 lufentl)alt Darin findet, fo wobls fur fich felbft, als ausb fir il)ren Sonmen in Die Falzen au legen; aber in holgemen Raiftgen, Die gut zu.

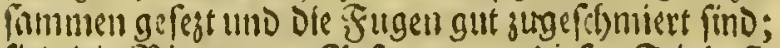

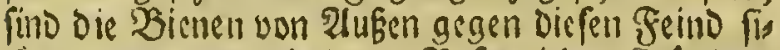
cher; und wenn die lecren Siofen jedes frrúljalye berausgenommen rerden, und Der Sclbmarm gientlid) ftarf ift, Damit Daf Die Siofen immer mit 23ienen beoseftefind, fo if von Somen nur wes 


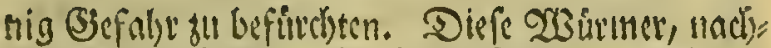

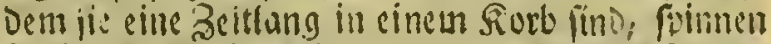
fid) in eine solbale ein and verdureen; in folgen.

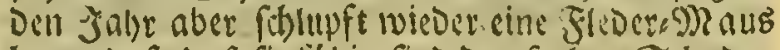
beraus, fo on B fie fályig fino oreufachen Shlodoen ;u thun.

\section{Sinitgen für \$ienen.}

Um die foeben befüriebenen 2 sirmer nus ben Bienen Etucten zubalten, forwoly! als wabre $D$ : conomie zu beobaclsten, find die folgenden be fartics benen Stócfe doer Fórbe, wie die Errfalyrung ges

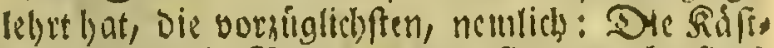
gen, (oder bie 23retter wobon fie gemaclst fino) misisen nur 5 Boll breit und 14. 3oll lang feun,

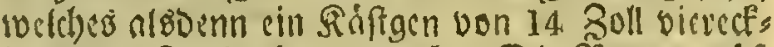
igt uno 5 zofl bodb, ausmad)t. Die SBretter mefs fen ungefelle brenviertel Boll bicf, ganz Dúrre sng finon abgehobelt feyn. Nindber weroen fie gut ges Dobodit (wo er weñ fie gut genngelt find, fo wite

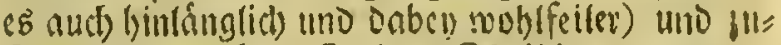

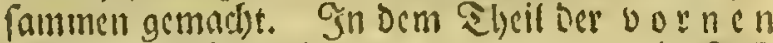
bin fommen foul, twirb alsoenn u $t e \mathfrak{a}$ ein foct) cingefigt, von ungefelyr zroen uno cin balben zod

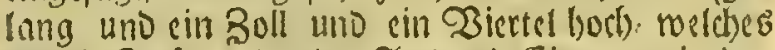

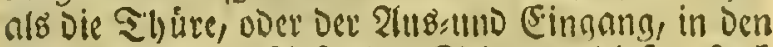
Stoct Dienet. 2luf jeber Geite von diefem Soct) mífill glvel) Eleine Seiften angenagelt feyn, fo Daf man swab bleclyerme S(t)ieber (nuf cinander) yor Das foct) madjen Ean. Sin Den Gmoendinen von

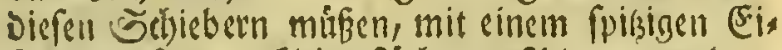
ren, ungefelle 30 El:ine Sóthev gefïlngen werden. siber Der austoendige Sthieber muf nut ein Sods 


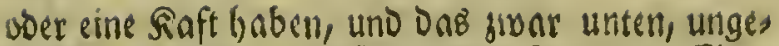
felor fo grof Dab zwel) Bicnen im 24 s = uno (Eiges ben einnnder pafiren Pónnen. Xuf Dex bintern Geite wird ein soch von ungefelyt fieben Boll lang uno Dres Soll brsit, gelasen, roorein eine Gjlab: Gdseibe getban wird. 2lufern an Diefer Gcheive miffen awev \&eiften angenagelt twerten, (auf jeber Geite eine) Die fo eingerichtet find Daf man cinen bojkernen Schieber úber oie Sd)eibe mact)en fañweldyer ar ein Eaden Dienes. Diefer Scbicber mus beftíndig zu brieben, Deun Die Bienen mollen su ilser Zrbeit ounfer baben; er follte aber Dods

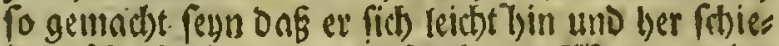
ben ldet, fouft toiro ex in feud)tem 2 Better unbes weglich. 2In Der obern Gelte Diefes fidftgens, mifien (aubivendig) ringơนm clne ausgelyobelte Seifte genagelt werden, die genall mit bem obeun Sianft gleids Fommt, und Derfelbe breiter wird, Das mit man benfelben sicbter jufd)mieren tan. 2Señ Diefes gethan ift, fo wáble man eing, weldbes das Dberfte feun foll: in biefer lafe man in vie oberfte Seite fi c be a seifen, osn ungefelse einem Boll Dicf uno eben fo breit, fo tief ein, Daf fie genau mit Dem Sianft gleid) Eonmen. Dicfe Eciften alifen fich von Der vorocen nad) Der lintern Geis te Des Riftgens erftecten, an welche Die Sienen ibre Siofen bebeftigen. Sebeb von Denen anoern Rifftaen befonmt nur ziven fold)e \&eiftem, Dic ien

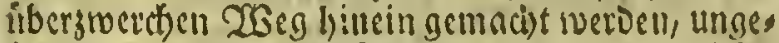
febr 2 uno cin bniben Boll von jeder Geite. [9]. Salafti, in feimem Dericht an bie Motriotiffoc (5e: fellfbaft von D? ?nyland' fogt, inan foll jedem Gifts gell 5 feiften von vornell nad) binten geben.] Der

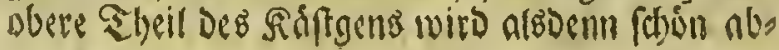


geljotelt, fo Dos man mit cinem binnen eifernen Dusut givifuen Den aufeinander gefésten Ráftgen Durd) fallersi fan, olste veft zil werden. Radblyer

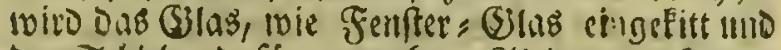
Der ङchleber Dafír gemact)t. -2UlFDenur mus man cin gutre Burt haben, von ungefel)r einem Bull Dice, fúr einen Decfel, Dicfer wiro Darauf nigige fobranbt doer auf fonft eille 21rt beveftiget, Duf er

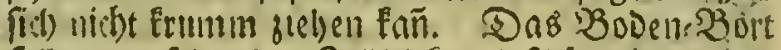

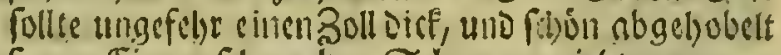
fel)n. Einem fobuacben Sdhwarm giebt man wilt

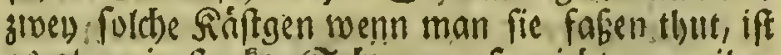
es aber cin finater Schtwarm, fo giebt man ibm Drè), und läkt fie nue nus Dem unterfen fiáftgen aแb und cin fliegen.

\section{Dir Nitben doet Bebentát von Den Schiebern an Denen Raftsen.}

Der Sebrands won Den zwew blecternen Sther bern, die nuf Die Slyire Doer Den 2lis" und Ein. gang foumen, iff, uns bie orgúrmer abullyolten; Dein ineil fie meturfens des 2rbends fommen, to mus iman jeden 21bend dic SBienen= Stocfe guma.

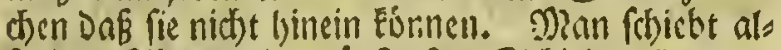
fo Des 2ibenos den áuferfter Sdhieber (Der nur cin Riftgen bat) linauf, und Den Snnendigen, mit Den Eleinen \&uftlochern binmnter, woourct) Die Bienen yom Erifiden gehalten werden. TSGill mon ifgnen in febe beisent 23 etter melye suft ges ben, fo fichiebe man audb die duferfen Echiefer an Den anderit Reiftgen linauf, Des Mioraens noev

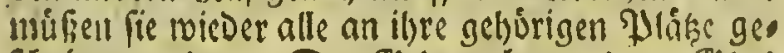
frioben werden. Der Sebrauds oon dem Eslas 


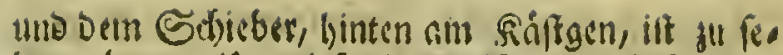
be!!, ob ctioa Unuegiefer Darin ift, oder ob fie il)e

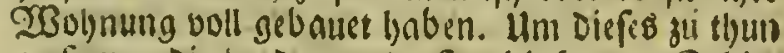
mufi iman die beyoen vorderfen blecbernen Scisie: ber gan binauf fobieben, tmo wenn man alsoenn Den Gdjieber binten von Dem Gilas fdrebt! fo tan man leidst felsen ob 20urmer oder fonft ltngegiefer Darin iff ooer nicbt.

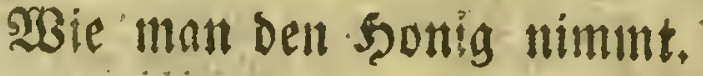

2 Genn man SBienen in Den vorber befohriebenen Sinftgen bat, fo Enñ man ibnen Den Sonig nebmen

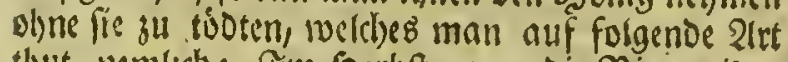
thut, nemidh: Gin Serbf, wenn Die Bienen ilje Schuler yoll gefaminlet baberi, faneide man mit einem Dunnen Drat (1Denn fie vier Rifigen voll getaut baben) eins Davon oben ab, uno faraube woer beveltige auf einige 2 (nt twie man es am bes. fern (i)un fan, wieder einen guten Decfel ohen sas rauf; boben fie funf ooer fetss soll, fo barf man ifnen zwoen Rifftgen oben roeg nelymen, und wenn es fich im Sribialse seigen follte, . Dafi fie noch sinet lleberfluf an Soníg baben, fo fann man ilsnen auf bie nemlishe P(rt nod) mel)r nel)men. - An eis jes Deg Enoe bon Dem Srat mit roelcbem man sie Siiltgen von einnnise fdineiden will, binde man cin Eleiner Solzgen, bamit man beffer Sant nel): Imen Bann. S(boenn nelyme man Joei) Uinne Meilfer ober Meffern und feetbe ein wenia in Die Fugen grvifiben dic Riffgen die man abfat)neiden will, auf beusen Seiten nur fo visl, dof man mit Dem Dinnen. Drat biriein $\mathcal{E}_{n}$, uno nlsoenn fdireis.

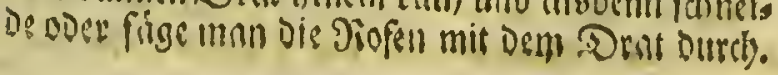


- Ehe man fie aber von sinnoer fatneidet, muf man eimen glten Ded el vorbereitet baben, Damit, weñ einer DnE Réftgen ablebet, ein allDerer gef(d)inino Denferben auf Die bleibenden Rifftgen thut. 2118 Denn geljet unn nit detn abgeljobenen Gíftgen ein wenig nuf Dic Seite uno ferset eB Dell verkeclses ten 20 eg (over DeB unterft íberft) nuf eine $\mathfrak{B}$ แnE oder ein fauberes 'Bord, nieocr; alsoenn flopfe man mit cinem leidteten Stecten ein wenig Dages gen, fo onk Die Bienen leraus fliegen; Herft folls te man aber ein fleines Jeller antínden, oder ines Sobnaf raudben fann, Der Eann fith Damit belfen, Denn mit Siaud ennn man fie am beften wegfreis Ben. Patblser frelle man fie all einen DunEéll Sort, fo fliegen fie nads uno nact alle heraus, Dods follten fie fo viel Sidt haben, Daß fie wegficgen fónnen. Der Dectel, Der auf Die zurice gevliebenen Sinfts Ben geoedet murbe, muk, fo balo als die SBienen

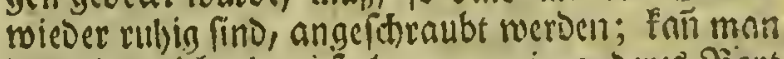
Daz aber nicht tlyun, fo lege man cin nnoereb bort oben úbergiverg Darnuf und befitstbere es und ber, fomiere Den Sianft gut mit fteifen Seimen, fo, Daf

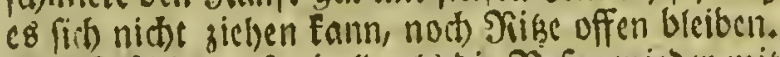
Om Sebjjaly, fo balo ald bie Fiofen woieder mit sBienen bedect find, und Das thiterfe Gisfigen bennabe voll gebaut iff, mus man ilinen wieder cin andercs unterftellen; unt fo fort, bis fic nicist melye biel arbeiten funnen. Siebt man ilynen vicl Sinum zun arbeiten, fo merden fie nur Defto frírfer, uno fotwodrmen nict)t fo oft.

\section{Befforeibung einer siappe, ic.}

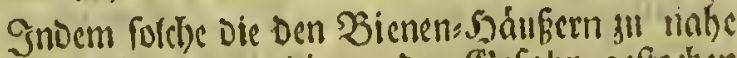
Fommen oose bounrulgigen, Der Gisfolye geffochen 
gu wetben ausgefegt find, fo ift eB nothuenoig firs? mit et:ous vorgufelyen, Daf man fid) ficber madben Pann. Man follte Destwegen Dicle XBollene Ricis Der anjilenen. Die Sánde fann man leidtt fis chern toeñ than ein paar dicte 2 Sollone oder Seder the Sano(d)uly ansielset; fiber Den Rouf aber folls te man cine linlánglich grofie Sinppe baben $\mathrm{tm}$ Das (Sefict)t uno Den ganzen Rovf zu bedecten; vor men follte eine Silab: Scheibe, ooer cin feines Sieb bon fsinem Drat Darin feon, Damit man felsen fañ, uno Dic Bienen Dois) nitjt ftedsen fónnen.

\section{Şom Schwårmen ber Biertel,}

und wie fie vom burdjgeben gebnlten werden funnen.

arbenn die Bienen balo fditodrmen wollen fo neisme man die folgenden Señieid)en wably nens lich wann fie viele Orat = Smmen machen (Die no(t) nictst gefdroármt baben) und bnben vide Ditifiggknger, bie fid) an Dem (2)tant in cincm grofien filum pen anbángen, uno nicht arbeiten ob fie fillon Sinum unio S3lumen genug baten; fo ift

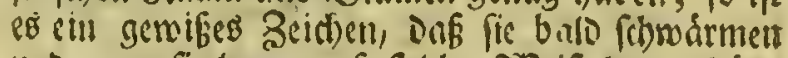
4.10 ivenn fie tange nuf foldse 3 Beife beraush in. gen, fo ift es gemeiniglitf ein Beiben daf fie ontets get!en wollen. Man muf alfo il)nen gut nufpafs

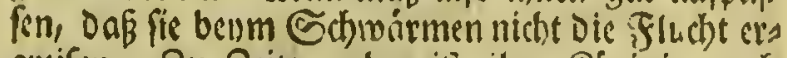
oreifen. Bu Bciten aber itt ilgre Sionigin noch)

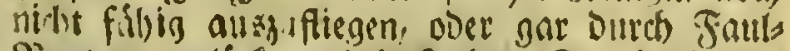
Sivut verunglifet, und in foldsem Sall bleibt Der

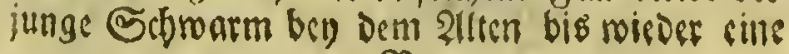
D 
Sonigin ba ift, weldses gemeiniglid) bev trev) 200 c)en Beit ntmmt, uno wenn fie vicl fraul: Brut bns ben, émnen fie manclymal Den ganzen Sommer nidbt fobrórmen. 2 Senn fie aber fetwarmen, fo mub man cin Ráfgen, wie auch cin abgebobeltco Bocn=2joro bereltet uno an Der Sando baben. 2Sollen fie fict) nicht feten, fo mus man mit cincm Jolyr ober tiner ongu gemadten Gprik̨e, wafer unter fie Sprisen, Doer Erose und Etnub unter fie wetfen, fo merben fie fich balo fesen, dab man fic fagen tann. So balo fie fid beftgefest boben, ipreite man ein leinen Sud) unter Den 23 aum, DDer fehe einen Sifd) Dabin und lege Das 230 den=2Bord Darnuf, alsoenn lege vier furze Stuctgen 50 olz nuf DaB̉ 23ocens:3orD, Die nicht weniger nls cis nen Boll Did, und ecfigt fint, fo DnB fie nid)t rols

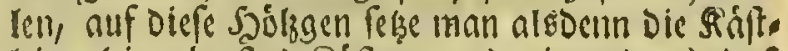
lein, (ein oberfes Sinftgen und ein anderí) Daß es unten offen it, Damit Die Biener alts und cins geben funnen. IIn Dem Unterften Sisftgen fojies be die bleckerne Gatieberlein hinnuf; an Dem Dberfen foriebe man fie alle berunter. Die Rifta gen mifien fodon grad aufeinatidel gefest febu; unto nacboell bie Bienen, fid) nlle gefert habell, nelime man einen blechernen Eimer, ein feines Gieb, oder fonft cin Sifhirr und fouittele ben Gcbivarm lincin uno fohutte dis cine Sańlfte nabe vor, die andere Själfte nabe binter die Silfte gen, fo werden Die 2 tenen fogleid) bingingelsen; wollen fie nid)t fogletel binein fo benege man fie

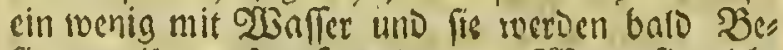

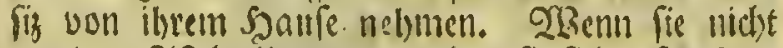
von Dem 2ift bleiben an weld)em fie fidl aefest bats fen, fo mü man fie auf bie remlidbe sist wiedes 
berunter boten; find fie nber felle freetig uno ges ben immer wisded zurfict donn made man ein wenig Rauci) mit sinem brennemoen ssmpen uni fie werden bato zu dem Gownarm gelyen. MRa muf aber $\$$ batst auf fie boben, uns wann fie ficis zu vid nufien an die Sirftgen fistu, fo mü ma!

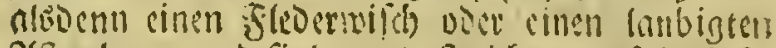

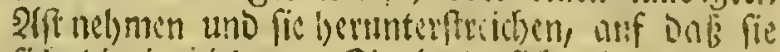
ficl) binein zicben. Co bull fict Die mebrften

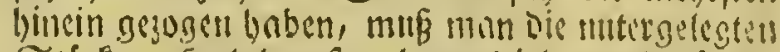

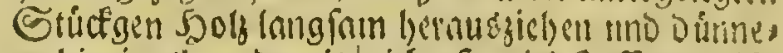
Ii binein thun bannit nidbt fo viel Deffinumg ift. croinn fie alle Darin find, nimmt mann alles Das runter berans, verfit)miert die gitize alle zu uno falicbt oie blecternen Gebieber bermnter, onb fie nut fo viel Juum baben, nlB gum 2lus uno Einges ben notbuendig ift. Dann nimmt man Jell Sd)warm mit Dem Sinftgen uno trágt fie auf Dit Ginno wo fie felen follen: bleiben Dann cinige surief, fo fino fie Dennod) nidst vertolyerl, benin fie gelsen gleid) wieder su bein siten toofie beramb famen. Gino fie unrulyig uno wollen nidlys Darin bleiben, fonclyme man clne Sjicks-Sianne mit $23 a f e c$, uno wann fie fict gu viel lyernubziclyen, fo muf man fie cin toentig naf madben uno fie wers

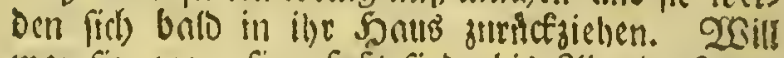
man fie, wem fie sefafit fino, bis 2 loents felsen laben fo mus man ibnen Sdbatten madben, Denn fonft treibt fie die SonnensSibe bernus. Lasenn fie abet bis 2(beno fteben bleiben, fo gewólsnen fich biele an biefen Dit unb ben nádyfen Sag werben wieber viel fid Dort befinder. 2 Benn man Dent jungen Edfroarm anf Den Stand ferget, mus man ilsn nidst aeben ben alten fellen nus weldbem fie 
Pamen, fonft gelen fie mandimal wieber zurnicf. Die firfigen in rocltese man fie fofen mill, múp fen fel)e rein und nicht ftin Eend oder fesimlicht foryn;

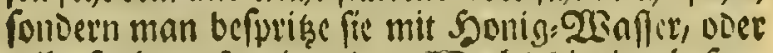
reibe faubern Sconig oder WBadt) limeill, befons ders an die oberften Srngs Sgiblgir. Shat maan aber

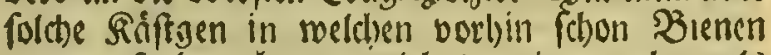
soarin, fo braudbt man nichts weiter fu thum als Diefelben rein zu balten; Denn von nllem was iman gebraudben Esann, ift iljnen nidjts fo angenel)m als Dasjenige mas fie fellef fammlen und cintras

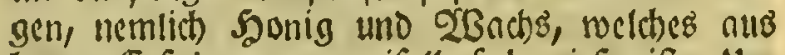
Innger Erfahrung unzweifelliaft benviefn iff. Un! Den gróften Piuken bon Den Bienen zul baben, folls te man fie nid)t oft fobroármen laffen, lino um oies fes zu verlyilloern, fogt ein berúlymter Ectsiftftels ler, foll man ilsnen, wenn fie ons stoeite oder orits te Mal fojnármen, Die junge Siónigin nclimen

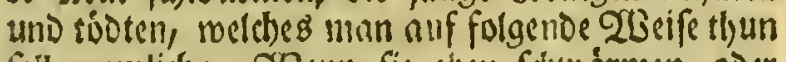

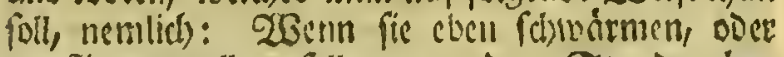
surffiegen roullen, foll man ju Dem Stano geben 3InD Dnieloft nuf Die Gónigin lonern, ullo wenn fie herank fommt, foll man fie fangen und tódten; 23110 Do fie grófer und lánges ift als dic andorn Sienen, fo eamn mon fie leid)t erfernnen, uno dies weil fie Esine Stachel bnt, uno folglich nidbta 34 firdsten ift, fann man fie leicht fongen roenn man

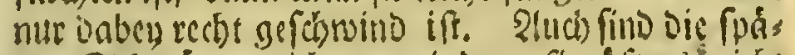
ten Schindrme micht gut, indem fie iftere nicts iberwintern fónnen. $2 B$ ent man fie niat bows

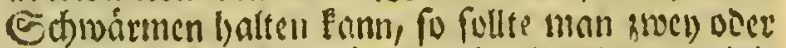
mebrere folde Echwisme mit cinander vereinis gen. Die alt' uno orseife mic diefer gethan wers Den mute miro in eineur nndern Enpitel angegerins meroen. 
Ea giebt viele Ecute die nllerlen cirle und abcrs glaubifide Dinge gebrnudben um die Dienen vom Durbigeben zu ballen, iveldbe alle vergebens uno umfonft fins; Denn wenn soir mit ciniger Ereatur sie unz ocr gutige (Sintt feluf umgelsen wollen, fo muisen wir zuerti ibre Satur und Eigenfehaft aubs finden und Danu fnun man fie ï son mel)rften Fof Kin zivilligen.

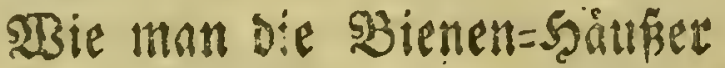

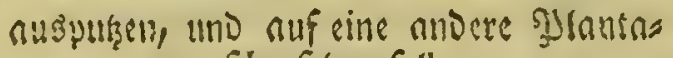
fibe feren fell.

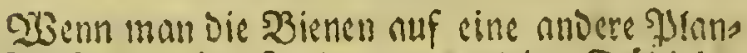
tafobe ftellen will, fo thue man co im Fruibjaly, sl)e fie ftere ficgen uno ibren Fisug beronnt fins. Einen neuen Edyxarm Eann mon am erftell sag roann er sefubt ift, binftellen wo man wili; aber einen alten Darf man in Sommer nidst berrieren: (Es gicbt selite bor(b) melnen fie ourften ibre Dies nen nuf feinen nnoern Tag berfellen oos suf cis

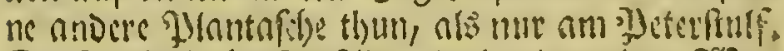

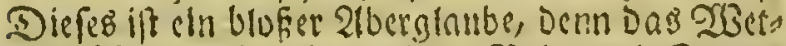
ter ift uftero nod) zu falt urio oce DBaden mit Eetnee bedecft. WBenn mon Denn suf cinen fo falten Ing cinen Bienen fiorb oder Santr aufmacles

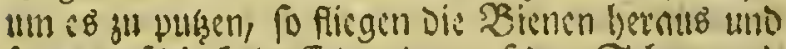
fallen auf Die Pnlte ErDe DDer auf Den Sodnee und fommen um; ift Das crostter noes fóbull, fo mis aen foldse, bie nuf sagen balten, fo tbun; mats follte aber Eeine Sienen derffellen fo lange ols $\mathrm{cs}$ nud) Schnce bat, ausacnomms!l man bialt fie z:!, Q2 2 


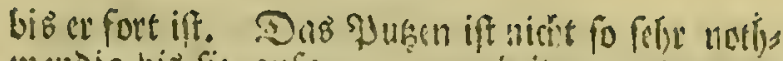

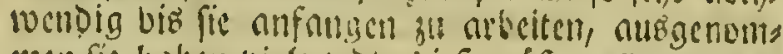
men fie briben vicle tobte, disfe miten nlle beraus? genommen weroen. Sound)e sente noer find fo unattrfom, onf fie Die lebensigen mit Den Sodten verberben Infen: Diñ im WBinter went die 2Bits terming felst falt int und Die Dienen Fnlt felsen, fallen ofters viel berunter gegen D 08 sodi uno fries re!t Do sufammen ouf eincu Silumpen, Dof Die, Die nod) leben, erflicten múfen. 20 cnn fie nter anfangen su arbeiten, fo mus man fleifig aus: puketh, uno Das Den ganzen (Eommet limüurd, oder wenigftens fo lange nlo fic nrbeiten; uno folle ten fie-pinmal 2 birmer bnbaty fo mus man ibs nen ifters cin foubces Sodenbre geben. TLin man aber fir geroif wiffu ob fie Griurmer lonben; fo lyebe minn die fieftgen Des Dorgens laugfan nuf uno felse, Denn unter Deut Sinnft uno auf Dem Boon, SBord fangen fie (Die Wínmer) nllemn! mit ibrer Derbectuma an. WOeil aber vicle \&els te furdotfam fino, fo fonnen fie cinen Desiffel uels= men ber einen Dicfen SBnllen lont, aber boeb fd arf ift, lno ftetle Damit unter Das Ráftgen nuf Den Boven:Poro und gervidste es fo loob in tie lible

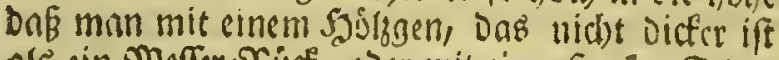

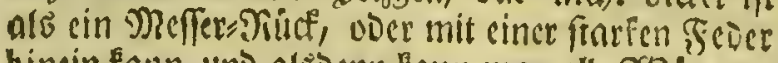
binein fann, und nlöbenn Bann man alle $2 B$ intmer, Die unter Dem Sinnft oder nuf Dem $\mathfrak{B}_{0 D}$ en find beraubfeliren; man mus abce die Sifftgen nicht fo boch lebendone bie Bienen leralis lónnen. 2uf diefe Art lann man fie fo oft auspusen n!b notlys toendig ift, olsne ibnen zu fdisosa. 


\section{Son Det Faul=\$stut}

WBann Dit Bienen Sautr.3rut baben fo frothen fie gerne nb, oder es wind wents oder nichts aus ii)nell. Solcte minfien gepulgt und die Siofen Dic faule Giungen entlyalten, minfen alle forgfistfig bero ausgenommen werien, fouft wenn etroas fauleg

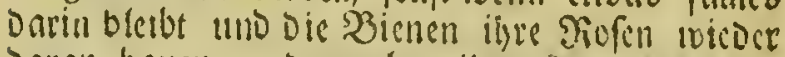
Daran bauen, und madjen illere Jungen wivecer

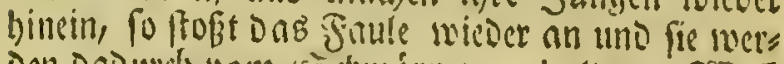

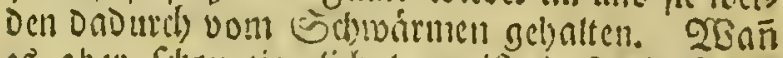

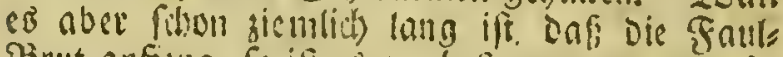

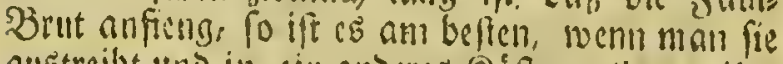

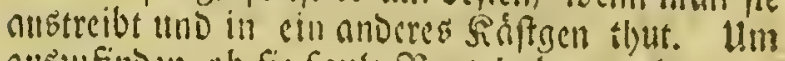
onts zufinden ob fie faule Brat boben, gelle man Des̉ Silorsens nalbe an tire sáftgen, fo wiro man

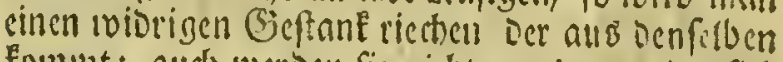

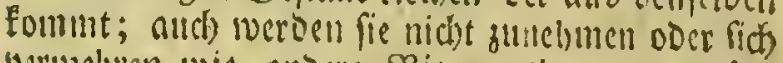
verturelyen tvie andere sBienen thun; uni láf: man fie fiels in, fo find fie Dod') nicmals gutt; uno

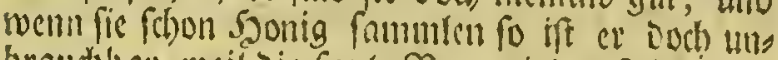

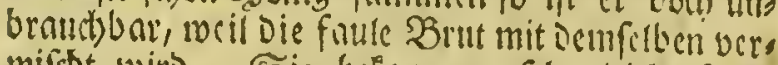

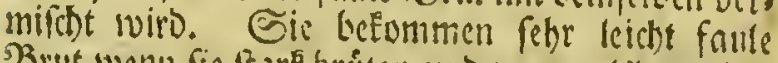
Bront wenn fic faure britten und wermn alsbern Eals

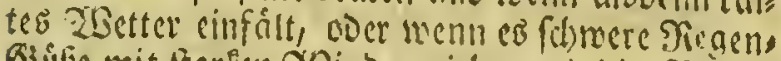

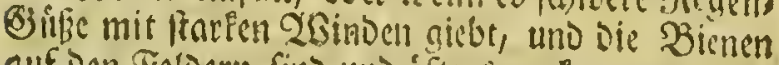

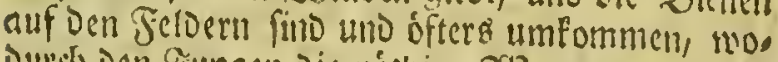
Durril) Den Gutungen Die núthige ZWartung entrifeen wiro und fie alsoenn auch veroerben múben.

\section{SBie man rie sienen aubreibet.}

2Genn man fie nubtriben mill, fo fan man

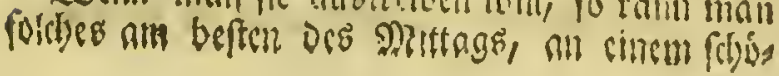


nen Tag thun, wann die Bienen sroffentlicite aurgefiogen lind; Drim nebme man fo vid Silfts gan alf Der Éclwarm bedarf, reibe fie gut mit

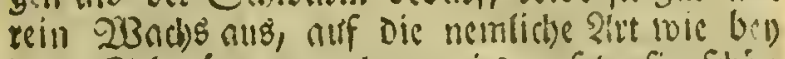
Dem Esbwarmen gethon wiro; féze fie fitsón

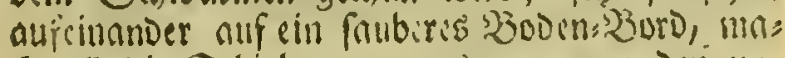
the nlle Die Solbeber fu, nแggenummen Den uns terfien, diefen lafie fo wett offen Duif die 23ienen gut pasiren fisonen; nlsoenn nimmit man Den Sil)narm Dell man nustreiben will, von feinem Erand weg und fegt die leere Senftach mit Dem

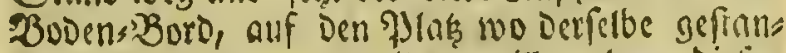
Den hat Den man austrtiben will, neljme bicien und trage ibne eine fleine Etredfe von Dem Etand soeg und felle illon Den verfelsten wer (oder DCB

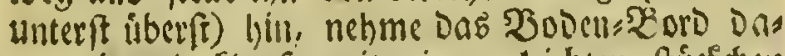
von ab und flopfe mit einemi leichten fticfeden

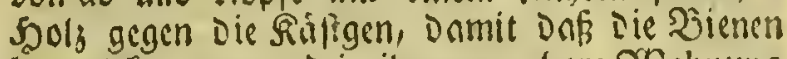
beraus fommen und in ilbre neue, (cere 250 olymm binflicgen too fie geftanden baben. Dian muß nbir vorficthtig feun DnE Die Rönigin nidt untecmt nod) verloren gebet; man mus fie alfo filye forgs fátig fongen und in die lecre 20 olynung tragen, Dann fie weif gar nid)ts von itsrem F(tig oder il) ret Selymatl wie dic andern Bienen thun, diss weil fie niemalb ausfliegt auв̧3enommen wann fie

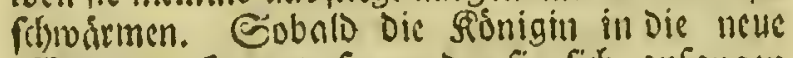
Wolgnung Eommt, fo werden fie fich) anfangell bu fammen uno hincin a ga gelyen. Eg iff nber ruch gut wenn man die Bienen bilitrágt, indem manch) inal vicle Sungen Dabey find die feinen Fing wifs fen, hefonders too man mormigte SBienen alisercis beil that, in weichen ifter Gunge find sie gar nitht fliegen lounen. $2{ }^{3}$ enn man fie hintragen. 


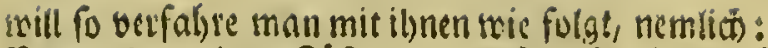
Dan uelyme lecre sinftgen und fore fie oben auf sietelben aus welchen man Dir Sienen treiben roll, uno flopfe immer ein wenig an Die unters ften, fo geben fie hinauf in sie Eeeren; mollen fie

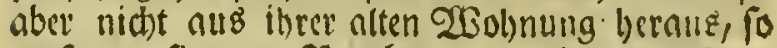
mute man fie mit Siauds berausglwingen. Jian

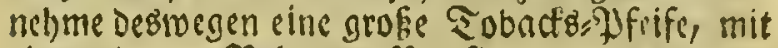
einenl langen Sidyr und fílle fie gans ooll bon tros cfenem ₹obact, bolye mit einem Ragel s Robrer ein Sod) unten in bie alte $2 x_{3}$ olynung uno ftecte onb Ifeiffens:iols binein, alebenu thue eill wes nig Seinmand úber Den Đfeiffens sopf, uno nelys tme Denfelben in Den Munto und blafe Den Sinud) bincin fo tocroen fie balo alle ausziel)en. Pinftatt yon Den leeren Ráftgen fonn man anch) Saubs? fte nelimen und úber ben Forb balten aus neldbem man oie Bienen beraustreiten mill, fo meroen fie ficl) Daran bángen uno alsoenn Pann man fie an ibre nene asolynung tracen. So bald die melse ften Bienen aus Den STufen fino, rimme man Dies felben forghfóttig heraua und trettet die sBicnen nlle meal Damit onf fie fich nithe melor oaran aufbals

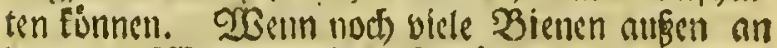
Der alten 2 zolynung find, fo trigt man fie an bie neue 230 hnuma und fiecicht fie mit etwas berune

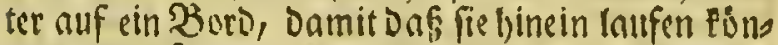
nen uns sufommen fomnten uno fo mettig verloren

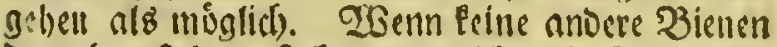
Dorueben fecl):n. Fo fann mand die mit SJonig vers fchmierter Riftgen Dnueben liegeri lnfen, onf fie Den . Jonitg beraufrangen Fónnen, fethen aber anz. Dere thahe Dahen, fo sehen nuds sic Fremoen bin und alboerin madben fie einander too. M)an Pann 


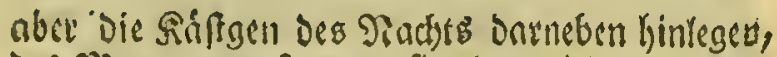
Des Mrorgens mus man fie aber wiever locgnel)? men, fonft Iernen die Fremoen DaDumely Das Siaus ben. Flud ift bu bemerten, oaf coenn man einers Sditwarm nustreiben till, uno nndete feclyen nas be onb sh, oaf fie uffers in bie lese 213ullnung eingelen uno fommen onourch um ilgr seben. In foltibem Fall mus man ein 20 oro zuifitien fie fiels len, Dns fie nidst fo leicht gufammen Eonnen; ibers baupt aber ift $\mathrm{eB}^{\mathrm{B}}$ nitht gut went fie ju nabe boy, einander fecleen.

Es wurve vorther fobon gemeloet dak man bev bem 2listreiben, bie Sionigin fangen mo in bie leere crsolnnung erngen fold; wenn man aber viele yon oett andern 2 Bienen liniber thagen thut, fo ife

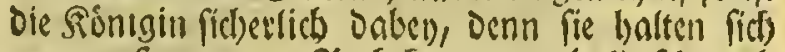
gerite zufammen. Ruaf ennn man balo felsen sb

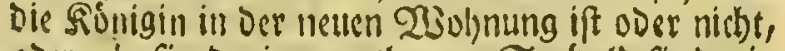
doer ob fie Dnrin gut thun. So bnld fie ontin bleiben uno gut thunt wollen, fo tauts)en fie ficts mit Dem foroern Elycil bennale auf Das $2 B o d e n=\mathfrak{B}$ ord, bnlten Den bintern Slgeil in die Soúle und fingen - Der Flattern mit Den Fligheln. "SSollen fie nbet nicl)t gut th) oo er Dnrin blcibet, fo faljrenfte nuf die 2irt toie benm Edindirmetl aus und cin uno bleiben nicht Darin. OVBenn man fie fafet uno fie baben ibre Rounigia verloren, fo muf man fie futben bis man fie fintect. Sont man fie gefunden

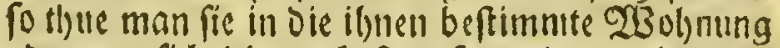
oder wo fich die inelyrfen fammlen; uno ine num

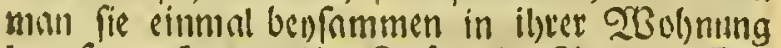
bat, fo mad)t man il)re Slyire oder ?l118\% und Ein. gang fleiner, fo toie ez Der Srbmarm bebarf.20 enn man sinen Sthwarm auf bie vorbefagte 
21t aแBgetricben bat, fo muß man ilbn cine Beits lang gut fintern, foult ibun fie nid;t gut uno bleis ben unr foltsi. onrili, gelien nuch leidit tod, toenn

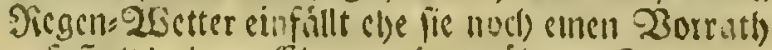
gefanclt bnbsn. (Einem mittelniabigen Sobwarm follte man ungefálsr wieder cin Peint Sonig ges beri, we(c) v: rbliten Daf Die andern fie itib) beffefslen uno ons Durd) rauben lernen. Diefer Weint Sonig muß unan ilynen noer uidst auf einmal gebcu, fornocrn nuf brew ooct viermaly nemlid), die crfte Radbt ungefón oie bálfte voer ben Dritten Sbeil Davon

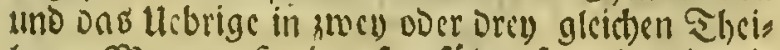
len.e Man mub noer forgfiltig feyn onmit oie Bienen nidts linein follen un! Darin too gelsen; um biefer su verhitin mue men cin Dimnes feis

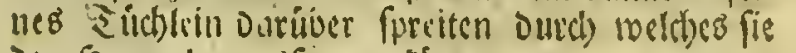
Den Sonig berauefrugen fónnen.

\section{:Bon Den Rạbern}

WBenn frtion die Bienen mit grofent Эied)t un ter vic coelfien Srcaturen.in ocr Sdjopfung, ges lechnet werien fontuch, fo baben fie Dodb, wie alle andite Crcaturen, nutb flute Fiebler, indem fie bem Etelsen fo ergeben find, Dafi fie, toenn

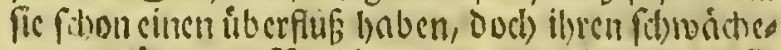
ren uno armeren ?Radborn alle ritruben tone fie baben, twenn fie cinmal die Obertinnd úber fie bes fommen; und fino destosgen mit einem mulberis

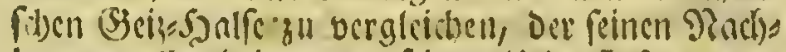
bat um nllez bringt tmu finen lleberfing gu vers grófern unD so od immer mad) melir trachtet. 2(boc

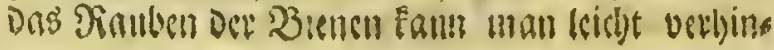


wern twenn man nicht ju fal)rldsig oose unerfafiren ifi. - J)?nu mus zum erften nuf die fobroaben

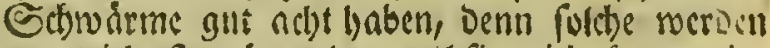
am leicbteften beraubt, well fie nicht fo gut im Stande find Die Siduber abzutlyalten; und menn man nlfo findet Daf́ fie von Siáubern angegriffen find, fo mus man ilyern 2H1s, und Eingang ver,

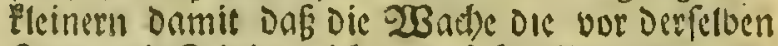

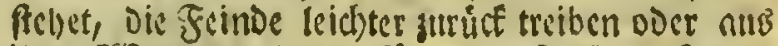

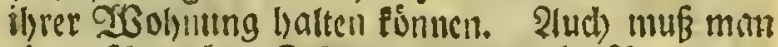
einem fditwacisen Echwarm nie su viel Sinum ges bell, Denn diefes ift iljnen fabádid) uno beforoert Die feindiche Einf\&lle. Wenn die Siñuber einmni solligen Eingaing in Den Sdywarm baben fo tnorden fie die Risnigin uno alsoenn hóret aller 2Siderftand auf, und die ifbermundenen (Finnolys ner laben fid) ungeftrift bernuben, belfen of, ters den noth úbrigen Shonig aufgel)ren, vercinis gen fith mit Den Feinden uno gehen mit ilyen in

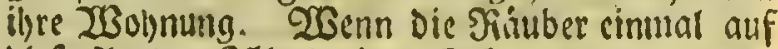
Diefe :urt Den Sñnhalt eines Schwarmes erlyalten haben, fo greiffen fie bald einen anvern an, und fo fort, bis onk Liebel allgemein miro uno gange Stínde zerfturt werden.

Um oiefes Uebel zu verbindern wird Der forgfirls tige Bienen: Eigner ein wad)fameb 2luge úber feis ne Bienen haben, von Der Seis an, oa fie im

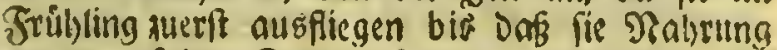
genug auf Den Fetoern finden; nach Diefer Bcit Eauben fie nur felten, biz Dag ifse Pialsung nuf Jen Fetsern wieder raber roird, tho alsoenn fans gen fie roieder an zu rauben, welches die nemlis che 2 urficht nutllig matit Die man im Fritl) ialye ju brobacten bat. T23enn man ju siniger acit 
Den sbienen Sonig nimme ooer futtert fie, fo folls te man fel)r vorfirtitin feyn und feinen Saonig bey Denl Grand verlieren, Denn Dndurch werden Dle Fifuber berbel) gezogen. Ez gicbt \&eute Die, weñ fie Sưrbe haben aus recichen Şonig genommen wurde, Diefelben grade vor Den SBienen: Stand legen, Damit Dak Die SBirnen Den Syonig Daraus nelinen follen; Dicfea follte aber nicht gethan wers Den, Derm 6 bicnct nur als cine Soctipeife um Feeinde bel) zu ziclen. Dicfe Kürbe oder Räftgen folle man werigfens 20 oder 30 Sd)ritte von Dem Stand wesfellen; uno mern man folwadbe Sdywarme hat, Denen man Dicfin Saonig zu bas ben winfdet, fo nel)me man cin Stút von einet Sonigs:Siofe, ooer frecthe cin wenig Solvig auf einen Spolyn, balte ilyn cine Eleine grseife vor bics

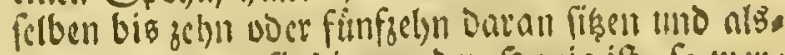
Denn trage man fie hin no der Shonig iff, fo wer" Den fie, inenn fie fatt find, heimflicgen und albs Denn find cn es auch Dic altocrn bafo aus und ges lent Dalyin bis oer Szonig all aufsegellet if. Shat

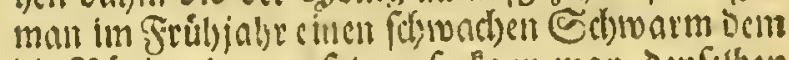
Die Siáuber bart sulfegctin, fo Eann man Denfelben sine Scitlang sulyaltenl uno Dabeu aber \&uft lafen Das fie nictit erfficfen: ooer man funn fie aud). in einen trocfenen Fellst, ooce fonft einen Dunfern Drt fectlen to fie nicht beunrulliget werden, bis Dic Slumen. Beit fommt Daf fie micoer arbeiten fónnen, fo fiumen ilynen die Sisuber nichts anbas ben. 2(mb) follte man úberbantut dic Sienen niche in Der Sonne frehen labeen Denn wenn Der Şounig warn wiro riechen ith Jic Saituber und machen Defto el)er einen Zingriff Darauf. Wlenn man aber

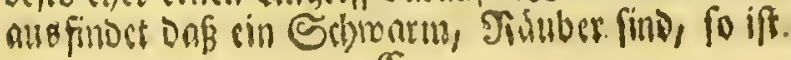


Eb gut tysun unan ifhen ein toenia Syonis nimme

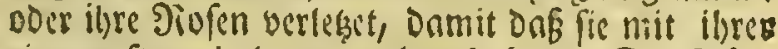
cignen Sanusbaltung zu thun baben. Die Siáus ber faun man alsigineen wenn man nuf il)ren Fing acl)tet, befonders wenn letive Szlumen find

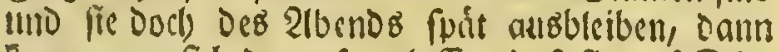
Eann man fich Earnuf verlaffen onf fie nuf Dies berey aus waren. 2uds fann man fie entoecfen, menn man g) iel)! nimmt und gehet an oen Forb Der geraubt rotro, uno fireuet ez auf oie, Die man Siumber zu feun glnubt, uno wo alfo nledenn bie melligten Bienen bineingelsen, Dort robnen bie Sifuber, eg mag nun auf Dem nemlidsen Stand feyn, oder auf Dem Stand einez Rachbarz; und swenn man alsoenn diefem Tindbbar Der Die Silus ber clgnet, Radjridjt Davon giebt, fo funtuen fols d) Mraabregeln ergriffen rocroen Dic Dnzu nuttlig

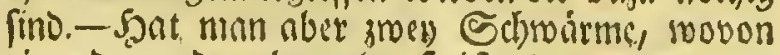
einer ben andern berallbt, fo if es gut tvenn man fie verwedb felt, nemlict), dell einen nuf bem and bern feinen Ylas fellet; Diefes gerirret fie und fie geben daz アinuben alsoenn auf.

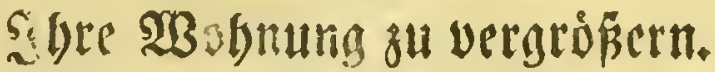

So bald als Die jungen Stbroirme einmal ans fangen su arbeiten, mus man aud) nufangen fie sull beobndten uno ausfinden wie biele fisftgen fie

- enit Sofen angefílt haben; fo balo alr Das uns terfte Siaftgen halb woll geboutet ift, muf man ibs thr mieder ein Eeeres unterftellen, reetches man

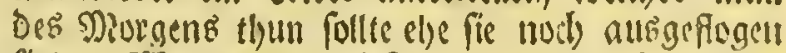
find. 2usenn man biefes thun will, fo thimmt

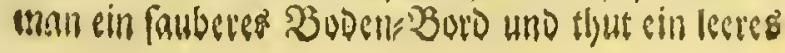


Rifftgen Darauf; nlstenn lafit man cine anbere Juerion ben Echwarm, Dem man feine 230 by nung vergrófern rill, nuflucben, uno ziclset Dns

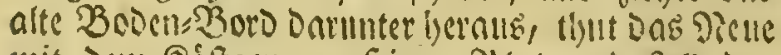

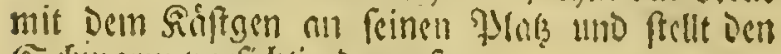
Sthwarm vorficbtig barnuf.

Sindboem man Den Grlimarm geforig gefort tho sic Sife verfdumiett bat, thut man bie Schicber nen Dem Sáfrgen welites yorlyin ons

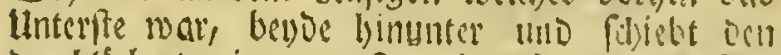
Durethlocberten im unterften binnuf, uno Den ciufs

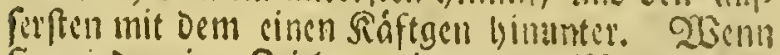
fie wieder cine Scittang, in gutem 26 setter gents beitet lyaben, uno das unterfte wieder beunabe bafber voll sebaut iff, to felle man ibnen allf Die nemlid)e Ant roiedst ein Eeereb unter; man

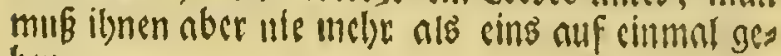
bent.

\section{BBie man jwè Sablwarme}

vercinigen foll.

Wenn man givel) Schrofrme mit cinander vers cintgen, oder cinen Ectiwarm DaraHö maben swill, fo felle man fie neben einnonoer, oer Deciet von cincm Baven wiro ntedocun abgenommen uno

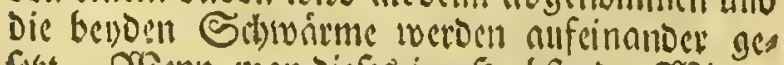

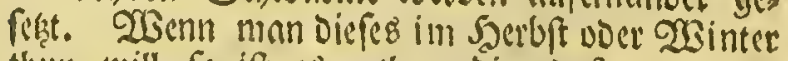
thiun will, fo iff es notllwendig, Dnk man alle Dem Sd)ronarm oce obch lyin foll, all bie lecren Siofen berausinimmt, fo Daß, wenn fie anfeinan, oer gereşt fino, feine Definung oder leere Etelle

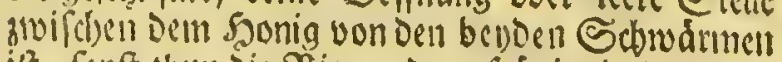
ift, fonft tloun Die SBienen Den Shinig in Dem lln 
terften SRffgen erft aufoelgen, uno wemn fie ifin aufgezelyt haben und ç fállt fel)r Ealte 233 ittes rung eill, fo fergen? fie ficts in oer oeffnung zmis fdien Den zroev) Sdlmármen auf einen Fiumpen uno fertern bor Saunger, restil fiton nod) cin lles

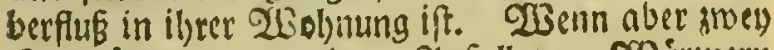
Sd)rodrme regen Dem anfall yon 23 irmern tniteinander ocreiniget werdell, fo bralicht 'man Diefes nidyt gu thun, Diensil in folctem Taul, Die sienen mit Sraud berunter getrieben weroen, uno Die Råftgen, Die 23 îrmer baben, gánglich ineggenommen rerden mifen.

Tuf Die obige Ilrt Eann man sloey oder melyo vere fdomadt) Ecbradume mit einanocr vereinigen;

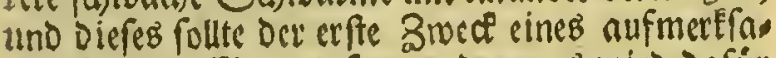
men BieneneEigners feyn; Denn es wiro Dafúr gel)alten, beffer zu feinn, eine grofe sinzaly! SBise nen in einem Stocf zu- haben, als ble Bahl ber

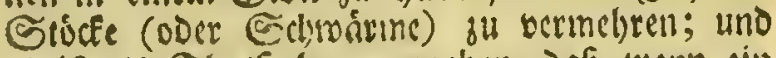
${ }_{C B}$ ift als Sbatfactle angegeben, Dafi wenn ein Stocf, ber 4000 Bierten in fich bat, $\mathrm{rech}$ : Jfuno Sonig liefert, fo liefert ciner Der 8000 b)at, vier uno a wangig Pfund. EB ift Desmegen Dem eigenen Intrefe cineo B̉ienene Eigs

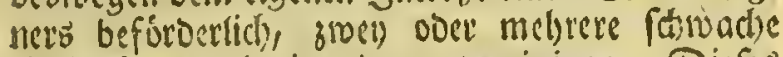
Edinnerme unit cinander all vercinigen. Diefes fnnn nuf oorbefingte sirt leicht getlan iocrden, bes

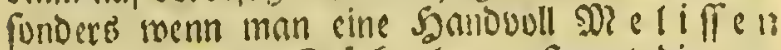
nimmt uno in Den Stoffen lyermm fretlet, Die man mit cinnmer vereinigen mill. Sulted Dicfes beo Fommen Dic ふienen alle ben memlict)en (Gerutb)

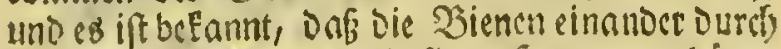
ben (Gserud) erFennen, ob fie zufammen gehoren Docer nid)! 


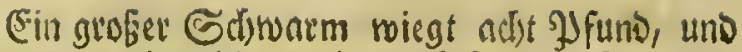
andere weniger bis zu einem Jfund. (Ein guter

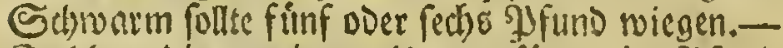

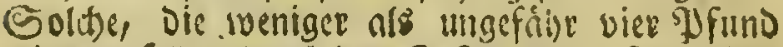
roiegen, follten Durd ben Bufak eines fothrondien Stbivarmes verfáret werben. Die Siróbe des sienen = Stocfeg (noer bie zufammen gefezten

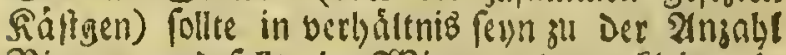
Bienen, untio follte im ZXBinter, el)er bll Elein, als 3"l grog fenn, cierbeil diefe Infecten melhr Wairme bon Nistben baben als ein gll grofer Gtocf geben Eann.

\section{Bon Dem $\mathfrak{B e r l u f t}$ Der Sidutigitt.}

(E) gefthiehat ofters, bas die Dienen ithre Fionis gin verticien, weld)es fin fie ein grofer Dets

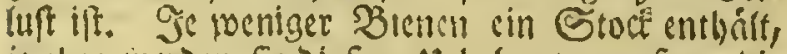
je eber werden fie Diefem $\mathrm{H}$ ebel unterworfen; die

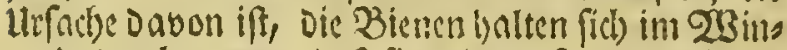
ter Dadurch warm, onf fie nillye zufanmen fiben, und ie gróferer Der Sifumpen ift, je wérmee wers Den fie fern. 23 enur man find d Daf cin Edbronam nitht arbeiten will, fo if es sfters nus diefer lits fadse; man follte Debrwegen Duesh Die Sdseiben an ben Sáftgen gut unthlucten, uno wenn man findet, oafe fie ilye Rounigin verluren baben, fo muf man fie olne Zeituerluft mit einem Sctiwarm vereinigen, welcher cine fionigin hat; fo wird if)re vorberige Traurigecit in frembe berwandeft, und fie fangen roberer an zu erbeisen. 


\section{Eimen Funftlichen S(bwarm}

zu machen.

M. Sonafisi, hált onfír, ona es beffer fels fünftidye Scbmorme zu madjen, als wenul man

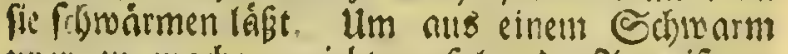

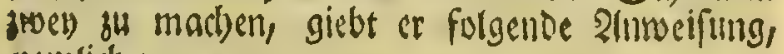
nemlid) :-

MRan nelyme einen Gtoct Der redbt gut mit 2Bienen verfeben ift, uno vier Siaffgen lyat, in cis nigen von diefen, befonders in den yroel) unterfen,

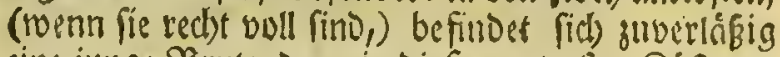
eine junge Brut; Denn in Diefen unterfen Riffigen, berindern fié gemúlhniglidj die jungen Sienen bon Der 2lurelia (ooer Hunollemmenbeit) aur Dollemmenbeit, weldyes ungefúlyr un Das En: De 2lprils Doer anfangs Ray, fentt findet; wenn nter Der Getoce nidit vell ift, fo gefd)irlbet diefe Werúnoerung nidlyt bis ausgangs May, oder mito ten im Sัแny. Lltm felbige Seft mus man cinen

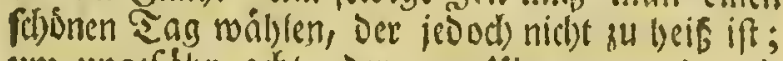
un ungefilyr acht oder neun ulue morgens, und elye die Biemen nocb febr ftare fiegen, inuk Der Stocf, in sivey getheilt werden, weldse an fols gende 2art gett)an weroen mus. Ziwifden oie swev oberften uno die glwey unterfen seáftgen, fees

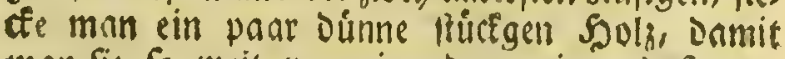
man fie fo reit von einander fruinge Daf man mit eimem Dúnsen Eifernen oder Deefingen Drat, Dle Siofen bon einnuber filyneiden Eann. Ein ree

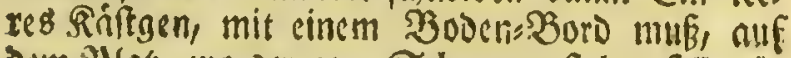
bem Jlas no oer neue Sobivarm felen foll, in Bereitf baft feyn uno man mus auch cinen gus teis Defel bey Der Santo baber; uno fo ges 


\section{(34)}

uig vouflanden iff, fo mus man fict) an anocec Nits ter menden. Shergu Enann man gutsı füben Ecis Der net)men, Der aủ fütien 2lepfel gomadst ift, soeld)en man auf eincun ftowacien Feuer su Mos

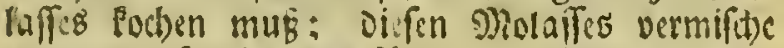
man mit Jonig, ungefílle sic ballfte oder sin viertes Elyeil; doer wenn man Eeinen Seider

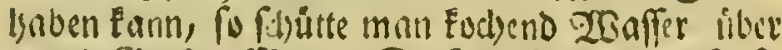

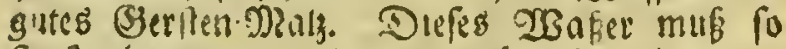
frare nogegogen merden als moglith, und wenn man ce auf die 2art nbtochet wie den Geider, fo Eartn man es itnen auf sie nentictse 2irt geben.

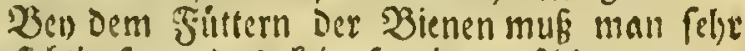
vorfidytig fevn, das pein Sonig verichittet miro,

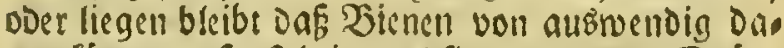

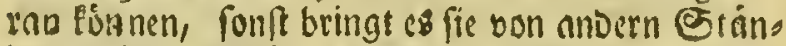
Den berben, welche Dadurch das Sinuben anf.ing gen. $t l m$ diefes zu vertindern mú man sillen Eleinen Srog madben, Der ungefdlyr adt, Boll lang, ein Boll tief und beu siven) Boll brcit iff. Fluf Dies fen seog mufi man von leidletem Syoly, einen Des cfel macten, Der ficl) in Den inneru Sbeil Defs felben pafen thut; in Diefen 2 ectel bobre man

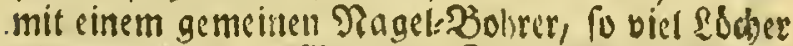
als man Pann. $\mathscr{X W}_{\text {Benn }}$ Der F rog mit Shonig ans gefíllt ift, fo legt man den Deafel Bnrauf, das mit Daß er fefrwinamt; Diefen Trog fetiebt man afsbenn in Den Stoce, uno zieht Den Durchlodsers ten Stbiever herunter. Der Deffel Der in Den Srog aemadtit werden muf, iff, um gu yerbins

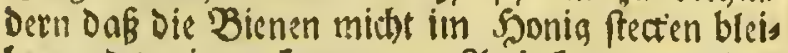
ben und 'arin umfommen. 2luch fann man Den Jonia auf einen Scller thun, welchen man in Den Stoct fetlet. Heber Den Syonig mus man 


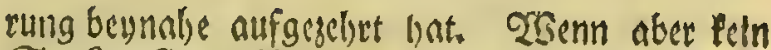
Stoce zu finden ilt, sem, wenn er fchon einen lues berflug bat, man Dodt tein ganges seiffgen net), men onef, fo mus man flum cime doer zlved Dio, fen nel)men, und mit fleinen Şólzgen in Den Pials

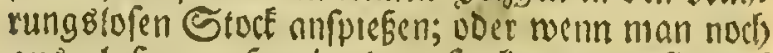
aubselnesenen Sonis bat, fo fonn man fie nuf bie nemlict)e sint fíttern, wie im vorigen Enpis tel nngenicfen ift.

Dian mus beforg fenn aużufinden roic viel leere Rúftgen cin jeder Etocf lont, indem man

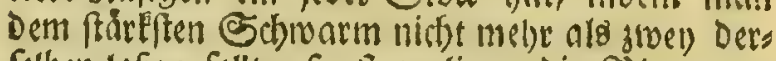
relben InBen follte, fontt verlieren die Bienen bu biel Reit in Der Sieinigung ibres SJnues, und nus Der nemlicten urfacle follte man ithen nudb ein fauberes Boden:Bjoro geben.

\section{Eine andere Wrt $\mathfrak{B}:$ enen = Etófe.}

Um die Fledermåufe, die ben Bienen fo gefályes lid) und foublich find, abzulyalten, mactse man für cinen jeo on Sthworm sin Rniftgen, Dag geras De folang und breit iff, wie die votilin befedricber

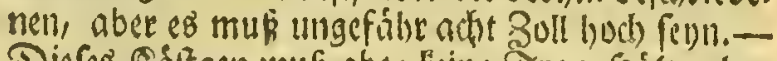

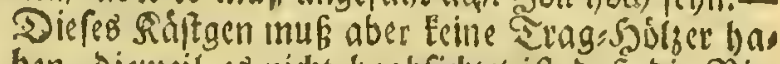
ben, Dietweil $c$ a nid)t beabridstet if nen binein ballen foller. 2Uuf dicfes Raftgen bes beffige man cinen gutten Decter, weld)er sin wes

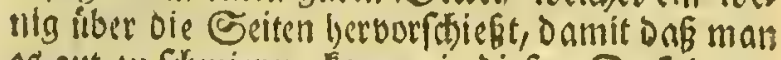
es gut zu falmicren fann; in diefen Dectel, nas be an Der yorderften Erite, gerade oberbnib Dem 2lub. und Elingang, madse man ein soct), weldtes nur fo grof ift daß die Bienen cinanoer gut im

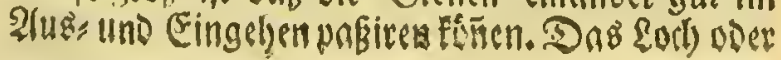


Rafigen, eine Scheite in Den hintern Sheil Defo filven sinmacben.

Saierbely if aber befonders au bemerten, Daf mail, wenn man forche Säftgen braucht, Den Bienen auf bie nemlide Ma lecre Riftgen unterftellen mufi, wie es beil Den nnDern Ráfts gen erfurderlid) iff, fonft bnuen fic in Oaz untes fee reenn fie in Den Sbern feinen Saum melse baben.

\section{Bon einem Bienen = Stand.}

Einer Der Saupt গুuncten in Der 23 nattung von Bienen, ift cin guter Stand. Diefer follte

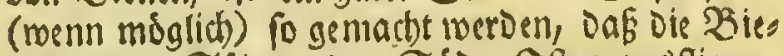
nen gegen Siben ooer Súb. Dften ausfliegell Esonnen; aud follte man Eeine Bienen binftellen wo fie vou Siaurt) doer (Sampf beunrubiget socrs Den, coer wo viel geflopft, oder fonft sermen gemadt roird; Deswegen follte man feinen BBics nen:Stano nalye an einem 230 olinbaus, Scheuer oder fonft cinem GSebáude, 100 geflopft, oder auf cinige Itrt gelármt wird, wodurd) oie SBienen ges ffort doer erfechúttert werden mógen, errichten. Man follte fee Desivegen in einen Garren frellen, Der

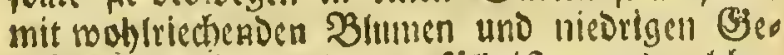
ftráudsen gefiert uno angefüllt if: aus welldyen Erferrn, fie einen Sheil ilyrer Plalsung bielsen fón nen, und wenn fie beum Sd wármen fich an bie

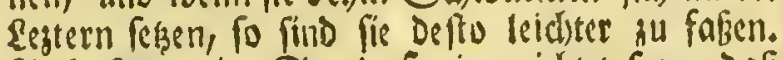
2luch follte sin Etano fo eingerid)tet fern, oafs man ote $\mathfrak{B i e n e n}$ gegen gu grobe Shise, Rílte obel

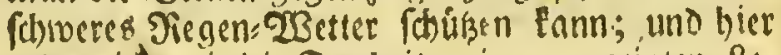
nelgne id mir bie Frevigeit meinen geresigen fes 
fern, Das Mrodel cines Stander anzuempfelien, Den ein gewifer Sarr in SBefts Sanover Tnuns Daupbin Sauntu, fur feine Sienen exricbret lyat. Diefer Stund befigt nidst allein alle diefe (sigen, fid)aften im bodffen (Sirade, fondern er if auct uolfommen binlánglich Die Fledermáufe aus den Bienen: Etodfen zll balten; uns weil in unfern Beifen jederman, Der SBienen bat, uber fie (Die Fleder:D2ture) flagt, fo ift es reid)(id) Die Diúlse nno fioflen mertl cinen folchen Gtano zu mad)en. Siefer Etand ift fo Didt gemad)t, Das feine ffeders: borbet und nn Dem bintern Sheil hat er eine Slyi: re Bnß man bincin geben Eanu. 2f Der vurocrften Seite, wo Die Bienen nus und ein fliegen, bat er grofie śdoen doer Sbúren, Die er jeoen 2lbend \$ll uno jeben Dlorgen wieder auf mait). In Diea fen Eburen bat er grobe blecherne Sdbeiben, in roldi)e er mit sinem fpikigen (Eifen, febr vicl flei, ne edeber gefdlagen hat, Damit Dab Die Bienen Euft haben, toann bie sliuren gugemndt find.Biefen Gtand lyat er fo grof gemacht, inf ct gwanyig Stodfe binein fellen tann; uno id) glat. be, inf Dicfes eine Der beften Erfinoungen iff, um Die Bicnen, forochl gegen ibre Frinde, nls

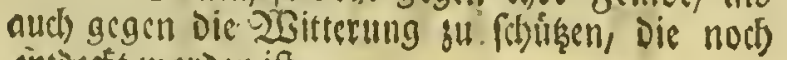
sintiect worden if. 


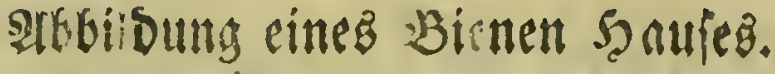

Folgenoes ift cine 2lboiloung uon sinem 2 Bieg".

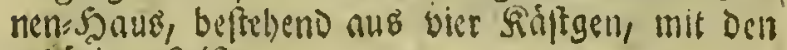
geljorigen suiften.

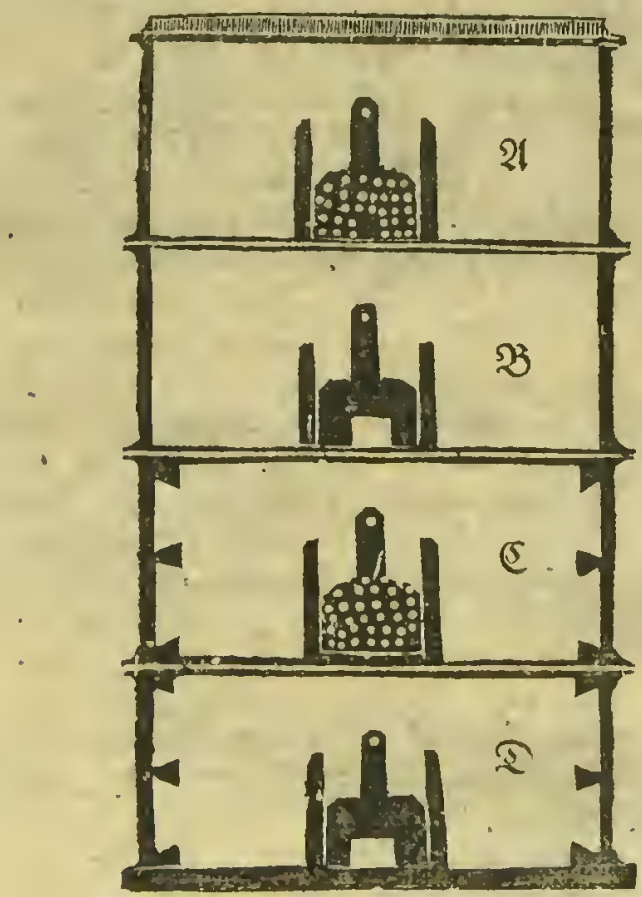

Das Unterfte von Diefen Raftgen, [D] zeigt ben fortoen Gebieber, mit einem sifftgen, Durd) weld)e die Bienen ntto uno eingelsen. Das

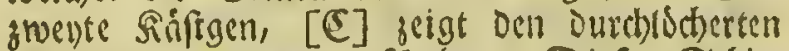
Schieber, berunter gefdoben. Diefer Edjis 
ber follee, wo er gegen Das Riftgen in Dem fo: liden (oder duserften Sobieber) fummt, nidst

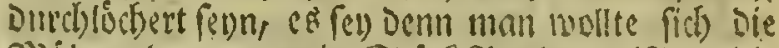
9) Rible nebmen um cin Ctúce Yapier Hwifdsen Die Cobicber an Den oberften Ritigen ju thun, onmit

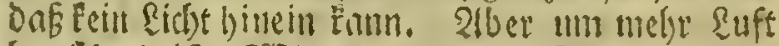

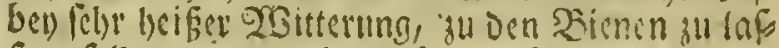

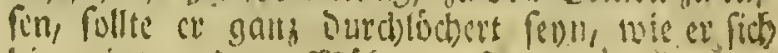

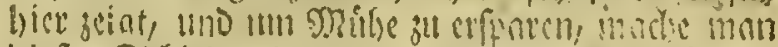
Diefen Gebieber, Demjenigen im Dbirfen sinfigen (2) gleich.-Das Dritte Sinftgen (D) tno Das vierte (अ) reprefentiren die fíftgen, zufanmen genagelt, obne Soboáles, aber Dod) mit seiften. Uln bie fisiftgen fo woblfsil zit madien als moglich, Fann man aucb die seifen reglafen. Gn bieferm Fall, follte man aber tleine bófzerne Siciber ooce

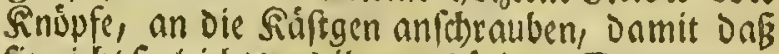
fie nidst fo leidt)t aus ibrer geborigen Etellung ges bradst toerden formen.

Folgenoes ift, fo biel als moglich, sine zes gliederte Jieprefentirung Der verfdiedenen Sbeis len von den vorbergebend befdyriebenen Sisfgen.

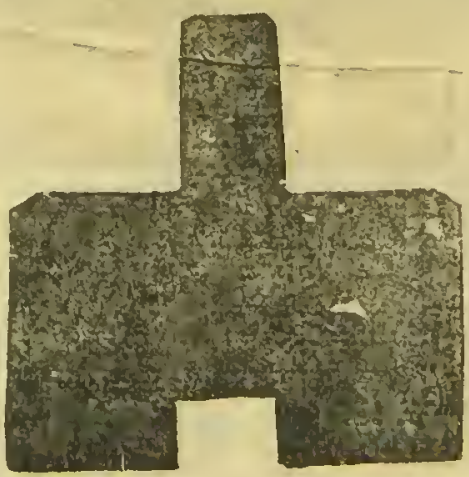


Der erfte Etid), reprefentirt oen foliden oder dukerften Echieber Durct weld)en Die Bienen ans. und eingeben. Der Stiel, oder be: J̧alt an Dies fom Cabiebcr, braucht nicht fo lang zu feun als nie er hier gezcigt wird, fomdern, wonn fich D D Dbled) niebt gut bafu fdieft, fo fann man nur Den oberfen Эianft unaefábe eincn viertel Boll leerum biegen fo iff Der Shalt nuch) binlänglid.

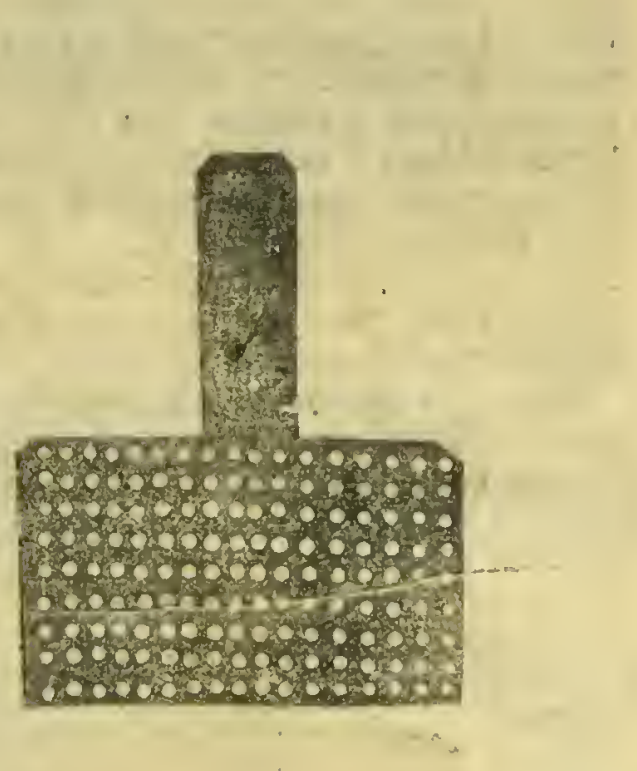




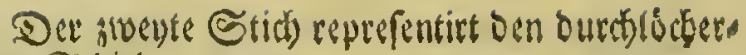
ten Sdieber.
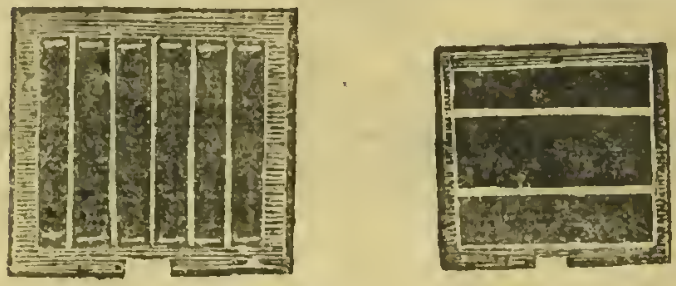

Der britte Stith geigt wie ong oberfte Rift,

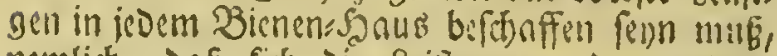

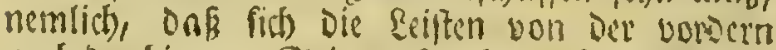
nacls Der bintern Seite ceffecefen mifen. 2(ued) ift $\mathfrak{e b}$ cine Teprefentirung von cinem Siefigen,

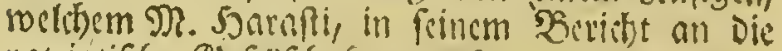

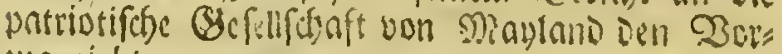
3ing giebt.

Der vierte Stid) reprefentirt cin unteuferer do

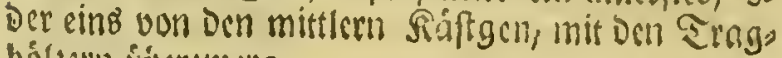
bólzetin ivierzroceg.

\section{Wie man cinten Scthourm} aus cincmi Strolys Sorb in dic Siffigentrciten fun.

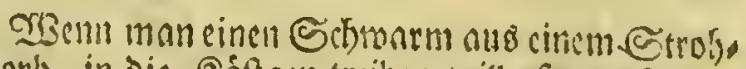
Sarb, in Dic Siffgen trciben will, fo mus man. 
cinen Decell hoben Der gróber ift nls bei SBoben yon Dem Sirnolys Furb; in Die Mitte van Dicfern Dedfel mus man cin Eoch cinfägen, wetcles um.

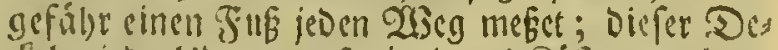
cfel wirb alboemn auf cin lecres Sinfitgen getl)an, weld)e zucrit anf ein BodereBorb gelfellt war: alebenn muf man jemand lyaben Der Den Gervlys Rorb nufluebet. Man nimunt num ong alte $\mathfrak{B} 0=$

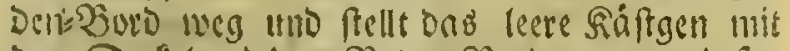

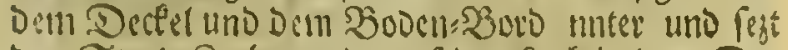
Den Strohy Sorb gernie auf Das Soch in Dem. De. cEet. Der Etrulys Sorb, no er nuf oem Dectel feclet, mus gut mit seimen verfdmiert twerden,

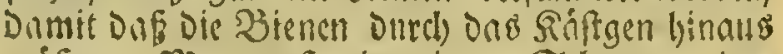
mifen. Pann mus abore einem Sdymarm, Den

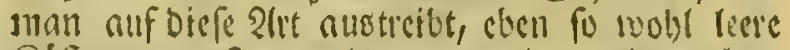
Siviftgen unterftellen als man an Den anoern thyn mus. 2luf Disfe glet léft man den storb bis in den Serbit fellen, uno wenn fie alobenn, fiven sidifgen voll gebanet baben, fo nimme man den Strolforb hinueg, haben fie aber biefelben nidt

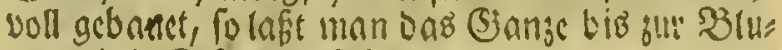
menjeit in Fribjalje ftelsen.

$23 \mathrm{cnn}$ man nun den Grwhysorb von ben Siffrgen wegnel)men will, fo mus man Die B̉ts

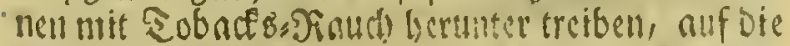
2irt wie nuf Scite 23 befibricben ift. Ele man aber Sinuth billetin blafet, fóbebt man oen Durd)s

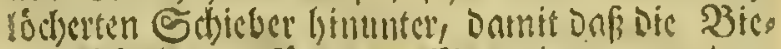
men nid) beraus foumen. Man nimmt nun ores

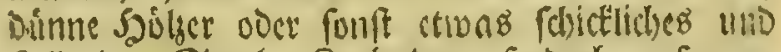
frellt Den Etroh s Sorb Dazauf, Dod') muf man

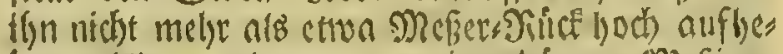

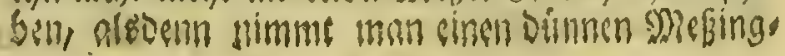




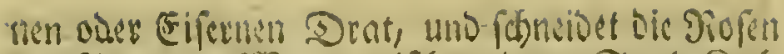

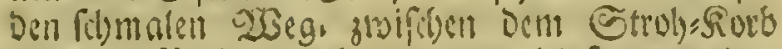
sno Dem Dord, Burd), und nun What man Ben

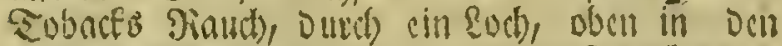
Strob)=Forb; fo balo nfel nle Der Siand unten ringsum nu Demferoen beriub Eommt, buret man

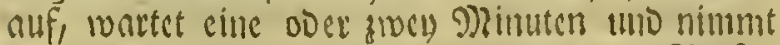
Den Strobs: Sorb binweg uno cine andere Ylerfon t)ut gefibumb cincu Decte! auf Die zurtict geblies benen Silftgen. Sollth einige Bienen in Dem weggenommenen Siorb bleiben, fo 1 mis 1 man fin nn cinen Dunfefin Drt fellen uno ibnen fo viel Sidtit

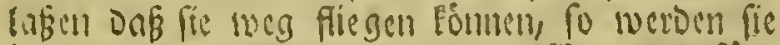

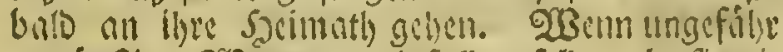
ungunftige OSBittcrung einfillen follte, che fie cis nen binlänglicben 2 orrath sefommelt haben, fo

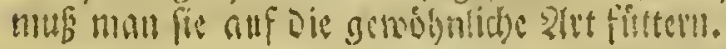

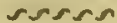 \\ \$gon bott Biench hergatwt.}

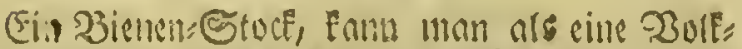

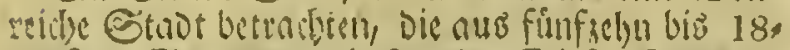
tanfent Eintudynern befteber. Siefe Etaot ift iin (iti) Relopt cime Simnblie, wo gut gcoroneter Tleif uno vollfandige Gilciblycit regiert. Die Siofen find aus reinem $2 \mathrm{~B}$ ad) ge nemadt, uno dic: nen als cin Mongajin fur the Tabrung uno Gun, gen biucin su thun uno Dariu gu erbalten.

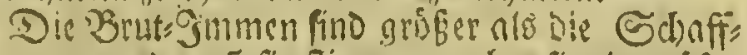

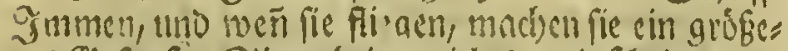
res (S) faufe. Gie arbeiten nidbs, uno forcinen nut 311 exiftiren, 3 m in Dem SBriten oer Jungsn bes 
Gilfflict) fu feon, für weldbe. Dienfefie boul Den ans Dern Bicnen erbalten werden. Slbe Extften ift jevodi nur von furger Doner; fie madhen ibre Er. fibeining im Frabjinter uno itn 2luguft ober ans fangs September, werden fie bon den andern Birnen too gemadit oder vertrieben.

Dor uften Beiten waren verfhiedene ?frten son Btenen ausgezerd)net. Eolumelln fiñit mit 2 irs

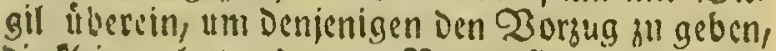
Die flein, glatt und guter $\mathfrak{R}$ ntui find: Der' grofie

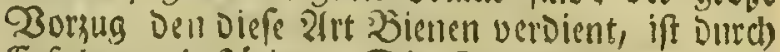
Erfal)rung beftitigt. Dis Gollaff: Smmen, fins Der Bablereid) Ite Sheil von der Trepublict. Eie baben oie 2fuffitht úber Den Etoce; fie fammilen Das Jibad;8 und Den 5onig; fúttern Die Gumnen; balten Den Stoct rein; trciben alle Fremolinge weg, und find befuffrigt ben allgemeinen 2 obls frand zu bifórbern.

Dic Bienen fojmármen nidèt, bis ilure gim!

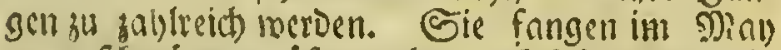

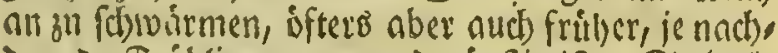
orm Derfrúlling warm und gimftig ift. Eo bald n!z "in Ed)marm fidi) gefegt bat, follte man fie fogleich fafen, fonft fliegen fie mieder fort. Weñ fie fiti) an. einen viebern 2fft borngen, fo faun man Denfelben nbfdenciden und neben die Gíftgen legen, in welche man fie fafen will.

Den Birnd Der Fislle Den die Bienen ertrngen ronnen hat man aoch nicht erfabren. Sn ben Ens ten Elyeilen von Suffilano, finiet man fie ofters

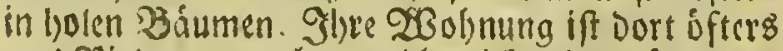
ถแร Siinden gemacht, weld) nictit visl wirme ges

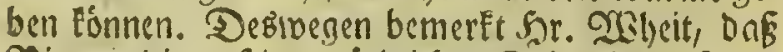

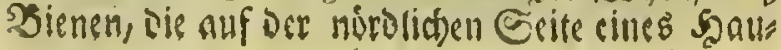




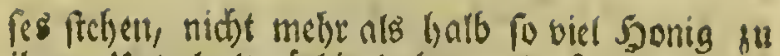
iberm tunterlynlt nithig baben, alB folde, Die in Der Sonne fetlen.

WBegen Der Sienten= Sinnigin mar man elsedem uneins, indem sinige belnupteten, fie bnbe feine Stact)el uno nndere behaupteten, onf fie eben fo ivobl mit einer Stndiel verfelyen (el) als mie die Sdbaffs Smmen. TRaturaliften baben nber diss fe Sadbe unterfudt)t und fanden, Das biejenigen dic berlegtern Dzeynung waren, redist hatten, ins Dem eg fich ber) Der Bergliederung einer Sisnigin jeigte, ons fie mit ermer Stadtel verfeben fino.-

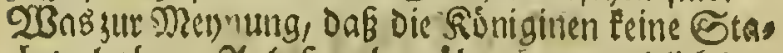
dyeln laben, 2Antne gab, ríl)rte vermutb)(id) Das

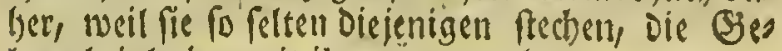
legentyeit l)nben mit ilgnen umsugelyen.

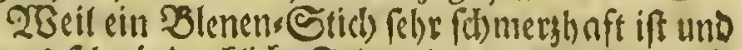

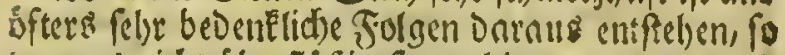

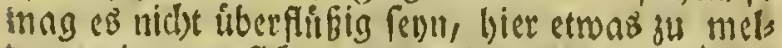
Den, wie man fidt) zil verlynten bat' wenn man von il)nen geftorten wirb. So belio ald man geftod)en wiro, mus man die 2lngel vorfidstiglids hernuszichen, benn wonn fie abbricht ober gu lans ge Darin bleibt, fo tlyeilt fie Defto mebr Ssiffit mit

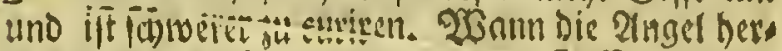

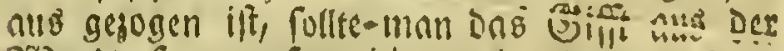
grzunde faugen, fo roiro wenig ooer gat kelne J̈nflomntion folgen. Crgenn nber Diefes verfâumit,

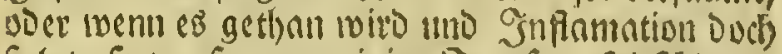
folgt, fo tropfe man einige sropfen Shiefthosus

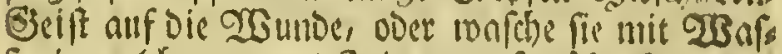
fer in roeldsem etwons Gnoigo aufgersist iff. Sant man aber feins your diefer Mrittellu, fo ift geners neB Sali die belte Cur,-20enn jemano fo unvor 
fid)tig peun follte, um cine Biente gu fellucfen odes

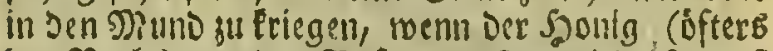
bel) Madt) ats Den Sofen geseften wiro, fo ints Das Sals aufgels fat und gefebludt rectoen, ift aber die 20 unde auswendig, fo macht inal fie feuclys und legt Das Snls Darauf. Drit nil Deir borlber befdyriebenen Curen mus inan anlyatten bis man findering bat.

\section{சck:0:39:}

\section{$\mathfrak{E} \mathfrak{e} \mathfrak{d} \mathfrak{l} \mathfrak{d} \tilde{p}$}

os

Ez if ber Sharacter Bug von mandien Deen fohen, immer an ote alten Gebranuthe zu bángen, ol)ne einige Derbefferungen in einigem Ding gu actern oder ilunen Slauben bely gu meffen. Eor de menfoben fino zu eigenfinig eine Probe von ei, ner uetten Erfinoung zu madten; fie glauben Der

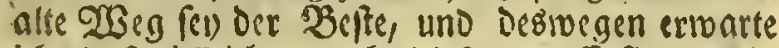
ich, Das vielleid)t mandbe Diefe nete Erfindung in Der $2 \mathbb{B}$ a r t ung Der $\mathfrak{B}$ ienen-s!b unnirs tilto

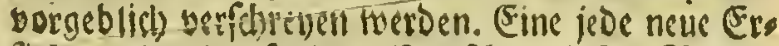

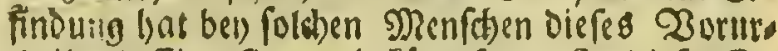
theil uno Eigenfirin zu befámpfen. In Diefer Evs finoung ift aber ein Srcibmittel, meldbes foldse Dienfclen fu einer Probe zmingt; ich meyne die WSirmer, welche fo allgemeine Berftorung unter Den Bienen angerichtet baben. Tus diefer lirfas the f(t)meicble ith mir ooch, Daf Diefe Erfindung allgemeiney und eljer erprober werden wird, ald 
überlyaupt mit andern Erfinoungen ber Fall ift. (2) vernuinfrig. Denfende, loiro aber bee Dem er.

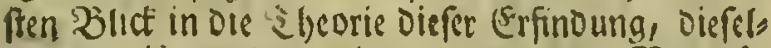
be gut beiben uns anu:lymen, woraus er Dergnile gen uaD Jrofit mit Buverfid)t erwarten fann.Es war Die 2loficht Des 2luthers, Die S beorie wos bon Diefer Birdlein bandect, fo Deutlict) uni bas bei) fo finz ż machen nls móglid), Danmit is cill jeber Der will, berftel)en Enan, uno aub biefer hrs

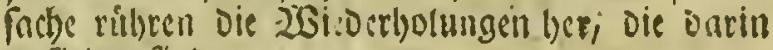
zu fino en find.

Die Gieftrien firr Bienen Die id) beftierieben l)as be find wabrfibcinlid) Den melyeften meiner sefern saus neu, fino aber Dennuch die beften die nod) is im (S) ebraud) voer befannt waren, uno 65 ift fels zroeifeslyaft, ob Diefelben ie serbeffert merden fónnen, obne die Ulifuften gróser zu mnchen als ibren Wertl). So wie Dic Séfitgen, zu machen befthrieben fitio, mag vielleicht inundser fith bor Der 2lusilnge firrthten uno fie Destoegen veradten. Riefes follte äber eeinen Einwano erregen, vies meil ein ieder felen fann, dos man fie auf den nemlictsen YSlan, auf wols feilere Rirt maden Eanu, als die bierin befidriebenen, nemlich, man braucht fie (roenn man fie felyr wollyfeil baben toid) witht mit Toved do zufammen zu matjen, fondern man unagelt nur, mit guten gefthnittenen Rhígil bies ferben zufammen uno láft aucl) Die feiften socg, fo bat man fie fo nos!ffeil als notluwendig iff, wern man Den PRusen betrochet Den man Darnus gielsen miro. E⿺ ift 3 u bemerfen, Daf im Embe Des 2Binters menn bie Sonne fidson fotent nie

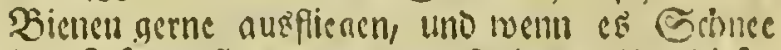
hat fo fallen fie germuter und perben, llin dieges 
gu berlindern haben ce vide Seute in Jebu

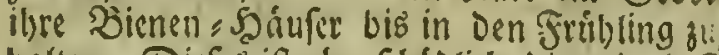
balten. Diefez ift aber fdádlid, Dieweil die 2 nen, wenn fie zu lange cinaefperet find, ilje Exii, mente in ibre 23 olsnung und bfters in oie Siofer. macten, uno im friibjaber fino oiefelken fo unecin Dá die Bienen lang mit ber Sicinigang Derfel, Ben zu thun baben, uno bfters reinigen fie oi fele ben nicht, wodurd) Der ganze Etoce in (S)efalye gefost wiro. Es ift desiwegen beffer, cinige we: nige umfo men zu lafen als Den ganzsn Sobmarm. in Sefglys bu fegen.

\section{ENDE.}

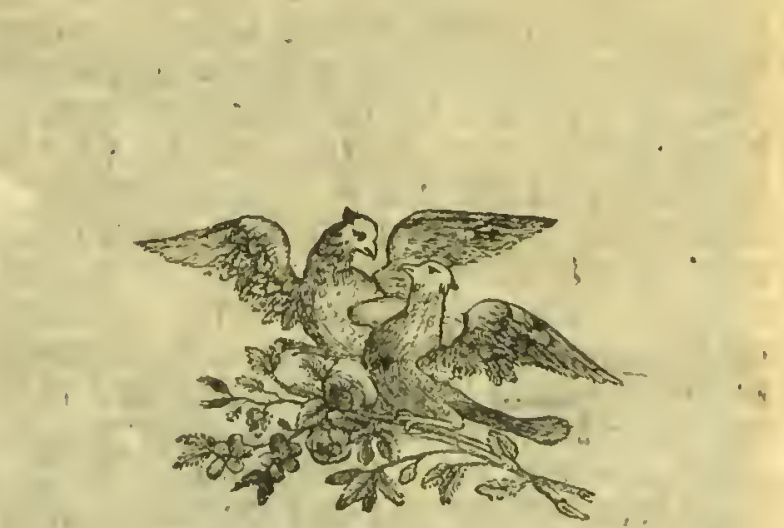




\section{5- 2) \{mmertung ủber Seite 12.}

Dieweil auf Seite 12 , in ber Befdreibung wegen ben Ediebern, gejagt roiro, Daf́ bie Sdbieber mit Dem einen fápigen jeben slbeud hinauf uno bie Durdldderten Edjieber herunte: geidjoben wera Den múffen, fo mag es nad) siner lueberfíd noth= rendig fenn, Dew unerfabrnen zu Shulfe zu fonme แ,

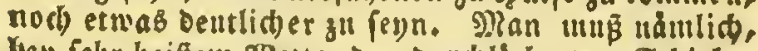
bey (eb)r beißem 23 etter den DHrdidberten Sdjiebet fo weit herunter fdjeben o aj́ nur eine $\$$ iene heraus

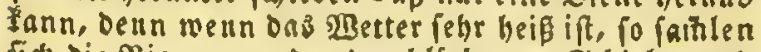
fid bie Bienen vor ben ourds lóberten Schieber unb balten ben andern die luft ab bas fie erfitien múf. jen. Im fribjabr und Epátjahr aber, wenn oab siEetter lubll ift, verfabre mall mit ben Sdicbern. zoie allf felbiger Eeite angewiefen ift. 2 lm beften if ez aber, um die flebtrmäufe abzuljalten, wenn mon jeben 2Lbend ein bunneg Tuds über Die Fáftgen hers zunter bángen thut, fo Ponnen fie Den Bienen nid)t beytommet. 












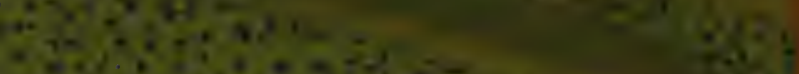

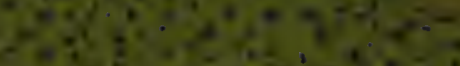

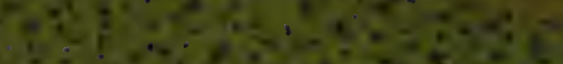

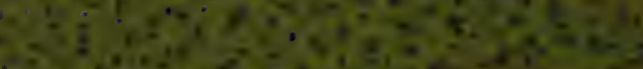

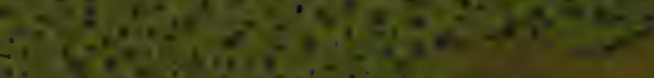

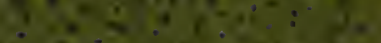

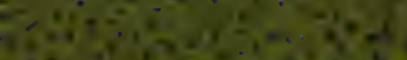

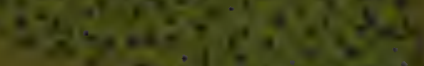

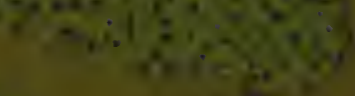

S.r.

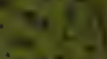

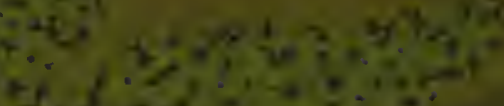

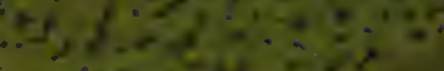

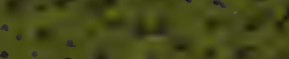

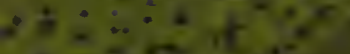

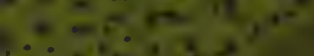

$\because E^{*}+4,+2$

$i+1,19$

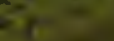

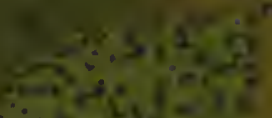

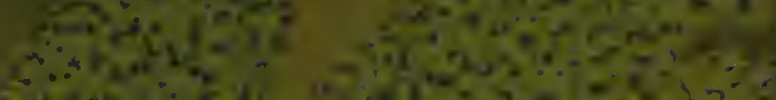

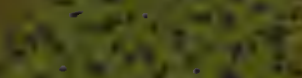

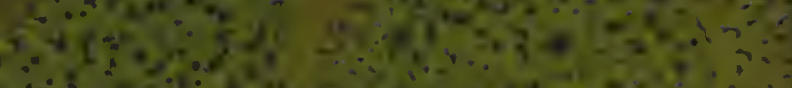

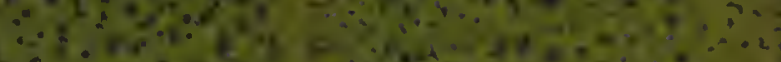

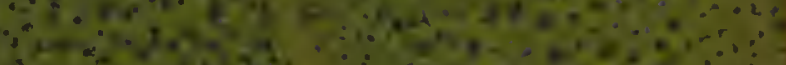

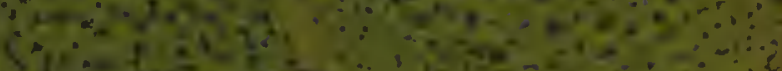

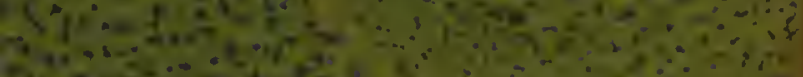

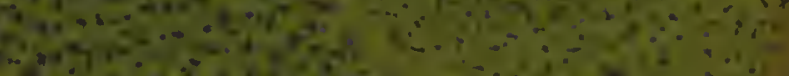

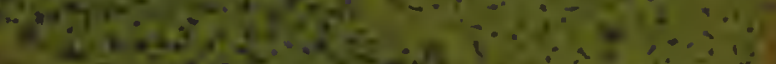

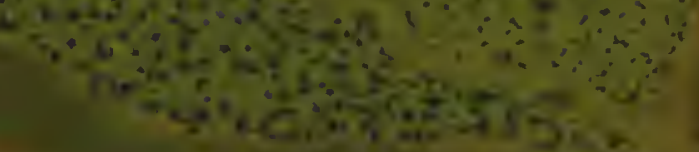

$=\because \because \because x y$

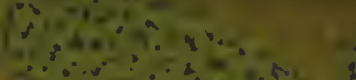

Cr.

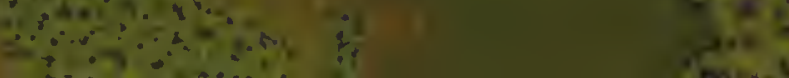

